\title{
Namur Open Architecture
} Die Namur-Pyramide wird geöffnet für Industrie 4.0

Die durch die klassische Namur-Pyramide beschriebene Automatisierungsstruktur ist bewährt und ermöglicht langjährige Betriebssicherheit. Allerdings fehlt den Systemen die Offenheit, neue Technologien werden erst spät verwendet und die Kosten sind hoch, was angesichts der rasanten Entwicklungen im Bereich von Internet of Things, Industrie 4.0, Mobile Devices und Big Data nutzvolle Innovationen verhindert. In diesem Beitrag wird das im Namur-Arbeitskreis 2.8 Automatisierungsnetzwerke und -dienste entwickelte Konzept Namur Open Architecture (NOA) vorgestellt. Das Konzept bietet sowohl für Bestands- als auch für Neuanlagen Möglichkeiten, um Anlagen effizient für kommende Innovationen zu ertüchtigen. Die Kernidee ist, die Daten der bisherigen Kern-Automatisierungswelt durch offene Schnittstellen wie beispielsweise OPC UA in die Systemwelt für Monitoring- und Optimierungsaufgaben zu exportieren und dabei die Kernautomatisierung weitgehend unverändert zu belassen. Alternativ kann über einen zweiten Kommunikationskanal direkt auf die bestehenden Feldgeräte zugegriffen werden.

SCHLAGWÖRTER Automatisierungspyramide / Industrie 4.0 / offene Schnittstellen / offene Architekturen

\section{Namur Open Architecture - The Namur Pyramid is opened for Industry 4.0}

The NAMUR Pyramid automation structure is generally accepted and supports long term reliability of operation. But these systems lack openness, new technologies are implemented with delays, and the costs are high. In view of the rapid developments in the Internet of Things, Industry 4.0, mobile devices, and big data, this obstructs innovations. This article introduces the NAMUR Open Architecture developed by the NAMUR working group 2.8 Automation Network and Services. The concept offers possibilities to enable innovative solutions for new and existing plants. The process control core remains largely unaffected. The basic idea is an open interface, e.g. OPC UA, between the existing core process control domain and the monitoring and optimization domain. Alternatively, a second communication channel can directly access information from existing field devices. 


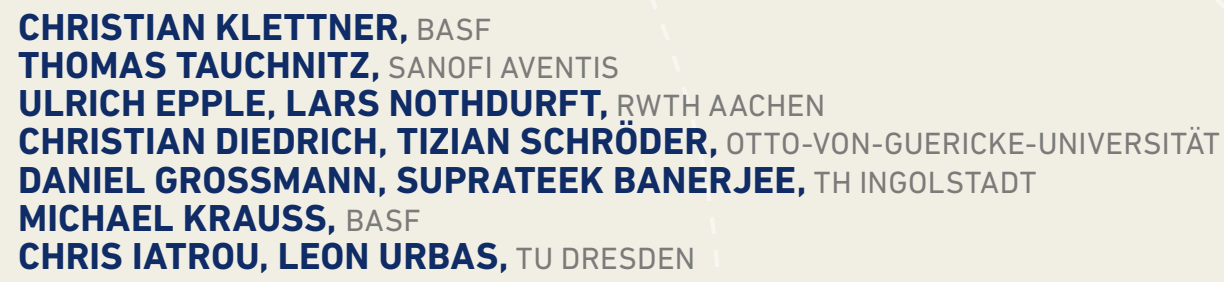

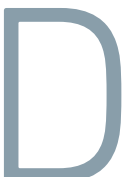

ie als Namur-Pyramide bekannt gewordene Automatisierungspyramide der Prozessleittechnik, auch Ebenenmodell genannt, ist seit ihrer Veröffentlichung eine bewährte und anerkannte Automatisierungsstruktur [19]. In der Feldebene werden die Sensorsignale erfasst und die Aktoren angesteuert. Die Prozessleitebene fokussiert sich auf die optimale Automatisierung des Prozesses, darüber sind die Betriebsleitebene und die Unternehmensleitebene der Automatisierung der überlagerten logischen Einheiten gewidmet. Auf der Feldebene sind viele Daten zu verarbeiten und die Anforderungen bezüglich Echtzeitfähigkeit und Verfügbarkeit hoch. In den höheren Ebenen wird verdichtete Information verarbeitet, mit Echtzeitanforderungen im Minuten- bis Stundenbereich. Durch den technischen Fortschritt werden - gerade bei kleineren Anlagen - die Funktionen von mehreren Ebenen in zusammenfassenden Systemen realisiert, es gibt also keine Eins-zu-eins-Zuordnung mehr zwischen der funktionalen Ebene und einer gerätetechnischen Implementierung. In den letzten Jahren wurde deshalb immer wieder die Auflösung der Automatisierungspyramide verkündet $[4,11,20,21]$.

Das geht an der betrieblichen Praxis vorbei, wo die Namur-Pyramide noch immer als hoch verfügbare, ausgereifte Automatisierungsarchitektur eingesetzt wird. Die so strukturierten Systeme erlauben der Prozessindustrie einen nachhaltigen Betrieb mit weit größeren Lebenszyklen, als die eingesetzten Komponenten von Haus aus mitbringen [16].

Doch diese für den Anwendungsfall in der Prozessindustrie optimierten Systeme haben naturgemäß Nachteile. Generell sind sie nicht offen, sondern geschlossen und monolithisch. Dadurch ist einerseits die eindeutige Verantwortlichkeit des Systemherstellers und -integrators gewährleistet, andererseits hat diese fehlende Offenheit mehrere gravierende Nachteile:

- Die fehlende Offenheit verhindert es, neue Technologien schnell einzusetzen, da auf die Realisierung innerhalb der jeweils vorhandenen Systeme gewartet werden muss. Häufig ist dafür der Austausch oder zumindest ein Upgrade der Systeme erforderlich, was erst nach Entwicklung der Systeme erfolgen kann und hohen finanziellen und Engineeringaufwand erfordert. Das hat beispielsweise den Einsatz von Feldbussystemen in bestehenden Anlagen erschwert.

- Die hohen Anforderungen an Anlagensicherheit und Verfügbarkeit erzwingen ein aufwendiges und dokumentiertes Vorgehen. Experimente nach der Trial-and-Error-Methode verbieten sich ebenso wie schnelle Tests von geänderten Methoden in Bestandsanlagen.

Im Ergebnis führt das dazu, dass die Prozessautomatisierung den Anschluss an die moderne Informationstechnik verlieren wird, teilweise sogar schon verloren hat. Und das wird aktuell als besonders schmerzhaft wahrgenommen, weil die IT sich rasant entwickelt. Es seien nur die Stichworte Internet of Things (IoT), Industrie 4.0, Cloud Computing, Big Data und Mobile Devices genannt. Hier werden mit enormem Aufwand von Staat, Universität und Industrie Methoden und Komponenten entwickelt, Architekturen entworfen und Dienstleistungen angeboten. Auch Unternehmen aus der IT-Branche, die bisher nicht in der Prozessautomatisierung aktiv waren, entwickeln Lösungen.

Während in diesem Bereich die ersten Ergebnisse sichtbar sind, gibt es nur begrenzte Vorhaben der klassischen Automatisierungsunternehmen für die Prozessindustrie, die Verbindung dieser neuen Technologien mit der klassischen Automatisierungswelt zu ermöglichen. Manchmal drängt sich der Eindruck auf, dass die Inselsituation der Prozessautomatisierung so lange wie möglich aufrechterhalten und ausgenutzt werden soll.

Diese unbefriedigende Situation führt dazu, dass unter den wirtschaftlichen Zwängen unstrukturierte Einzellösungen entstehen. Beispiele sind:

- Ausstatten einzelner Feldgeräte mit Sendern zur Funkübertragung von Messwerten - statt diese Werte vom PLS abzugreifen,

- Vor-Ort-Änderung von Feldgeräteparametern durch Bluetooth-Schnittstelle - statt durch das zentrale Feldgeräteparametrierwerkzeug, 
- Auslesen der Messwerte durch eine zusätzliche Schnittstelle des Feldbusses - statt vom PLS, Archivierung auf externen Datenbanken statt im MES.

Die geschilderte Situation - nicht nutzbare hohe Innovationsrate außerhalb der Prozessautomatisierung, fehlende Offenheit und unstrukturierte Einzellösungen - kann nicht im Interesse der Betreiber verfahrenstechnischer Anlagen sein. Deshalb hat die Namur Anfang 2016 innerhalb des Arbeitskreises 2.8 Automatisierungsnetzwerke und -dienste mit der Konzeptionierung einer offenen Systemstruktur, der Namur Open Architecture (NOA) begonnen.

\section{ZIELSETZUNG VON NAMUR OPEN ARCHITECTURE}

Zielsetzung der Namur ist es, die bestehenden Automatisierungsstrukturen so zu erweitern, dass eine flexible Umsetzung von Industrie 4.0 in der Prozessindustrie, sowohl für Neu- als auch für Bestandsanlagen möglich wird. Für diese Erweiterung gelten folgende Anforderungen:

- Die neuen Methoden sollen optional und additiv zur bewährten Automatisierungsarchitektur einsetzbar sein. Sie sollen diese also nicht ersetzen, sondern erweitern.

- NOA soll auf bestehenden Standards aufsetzen und dadurch offen und unabhängig von einzelnen Anbietern sein.

- NOA darf die Verfügbarkeit und die Sicherheit der bestehenden Automatisierung nicht einschränken oder gefährden.

- NOA soll schnelle und kostengünstige Implementierungen ermöglichen.

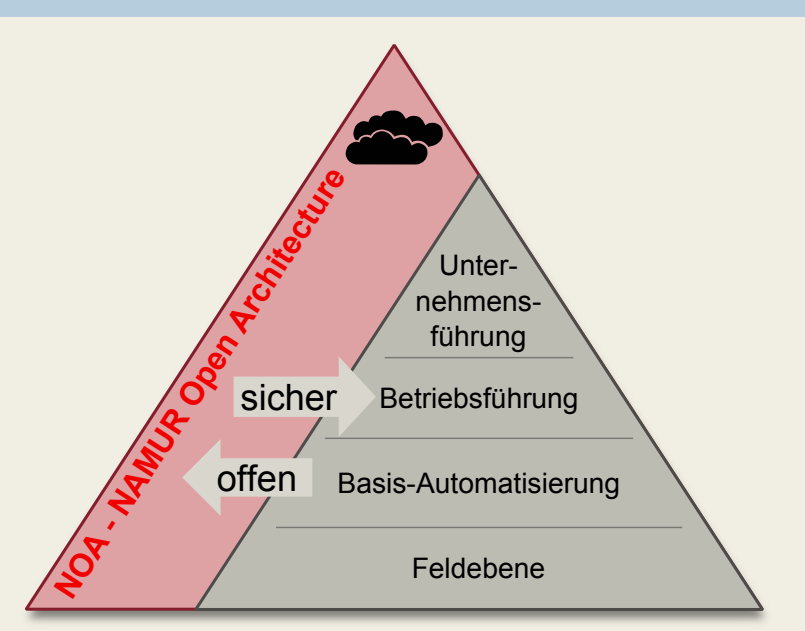

BILD 1: Namur Open Architecture als Erweiterung der Automatisierungspyramide
- NOA soll auf verschiedenen Ebenen an die Automatisierungspyramide andocken können, also wahlweise auf Feld-, Prozessleit- oder Betriebsleitebene.

Die Zielsetzung wird in Bild 1 dargestellt. Rechts - grau hinterlegt - ist die klassische Automatisierungspyramide. Daneben - rosa hinterlegt - ist die Industrie4.0-Welt, symbolisiert durch die Wolke der Cloud. Die Anbindung NOA ist sowohl offen als auch sicher.

\section{2. ÜBERSICHT NAMUR OPEN ARCHITECTURE}

NOA unterscheidet drei Bereiche, wie in Bild 2 gezeigt. Der graue Bereich ist die Kernautomatisierung, die den sicheren und zuverlässigen Kernbetrieb der Anlage gewährleistet. Daneben werden sich Bereiche stärker ausprägen, die heute teilweise schon vorhanden sind und Aufgaben zur Optimierung und Überwachung (Monitoring and Optimization, M+O) übernehmen. Hierbei handelt es sich um Aufgaben, die nicht für die Kernautomatisierung eingesetzt werden und somit keinen direkten Einfluss auf die Anlagenverfügbarkeit haben. Diese Betrachtung ermöglicht eine Differenzierung in den Anforderungen, sodass ein Raum für neue innovative Anwendungen entsteht, ohne in Konflikt mit den Anforderungen den klassischen Automatisierungsaufgaben zu kommen.

Im Bereich Plant Specific $\mathrm{M}+\mathrm{O}$ finden sich betriebsnahe Applikationen und Services, wie beispielsweise Advanced Process Control oder Alarm Management. Bei Central $\mathrm{M}+\mathrm{O}$ sind anlagenübergreifende Applikationen und Services, wie Reliability Center oder Plattformen für Advanced Analytics für prädiktive Instandhaltungsanwendungen zu finden. Im Zuge der fortschreitenden Vernetzung und Digitalisierung ist ferner eine firmenübergreifende Vernetzung über Central-M+O-Komponenten zu erwarten; beispielsweise einen gemeinschaftlichen Serviceansatz zwischen Anlagenbetreibern und Equipmentherstellern. Der $\mathrm{M}+\mathrm{O}-$ Bereich bildet somit eine offene Grundlage für neue Applikationen, Services und Geschäftsmodelle im Sinne von Industrie 4.0.

Die Einordung in die Bereiche muss applikationsspezifisch erfolgen. Eine entsprechende Metrik zur Einordnung wird durch den Namur-AK 2.8 erarbeitet. Der Charme von NOA liegt in der Anpassungsfähigkeit in bestehende Anlagen. Durch offene Ansätze in Koexistenz mit bewährten Strukturen können bestehende Anlagen nachgerüstet werden, um neue innovative Anwendungen zu implementieren.

Für die klassische Automatisierung bedeutet die konzeptionelle Entwicklung von NOA jedoch keinen Entwicklungsstopp. Auch im Bereich von Core Process Control sind weitere technologische Entwicklungen, wie zum Beispiel eine offene Schnittstelle zwischen Feldgeräten und Prozessleitsystem, sowie die Weiterentwicklung von IP-basierten Kommunikationstechnologien für die Feldebene notwendig. 


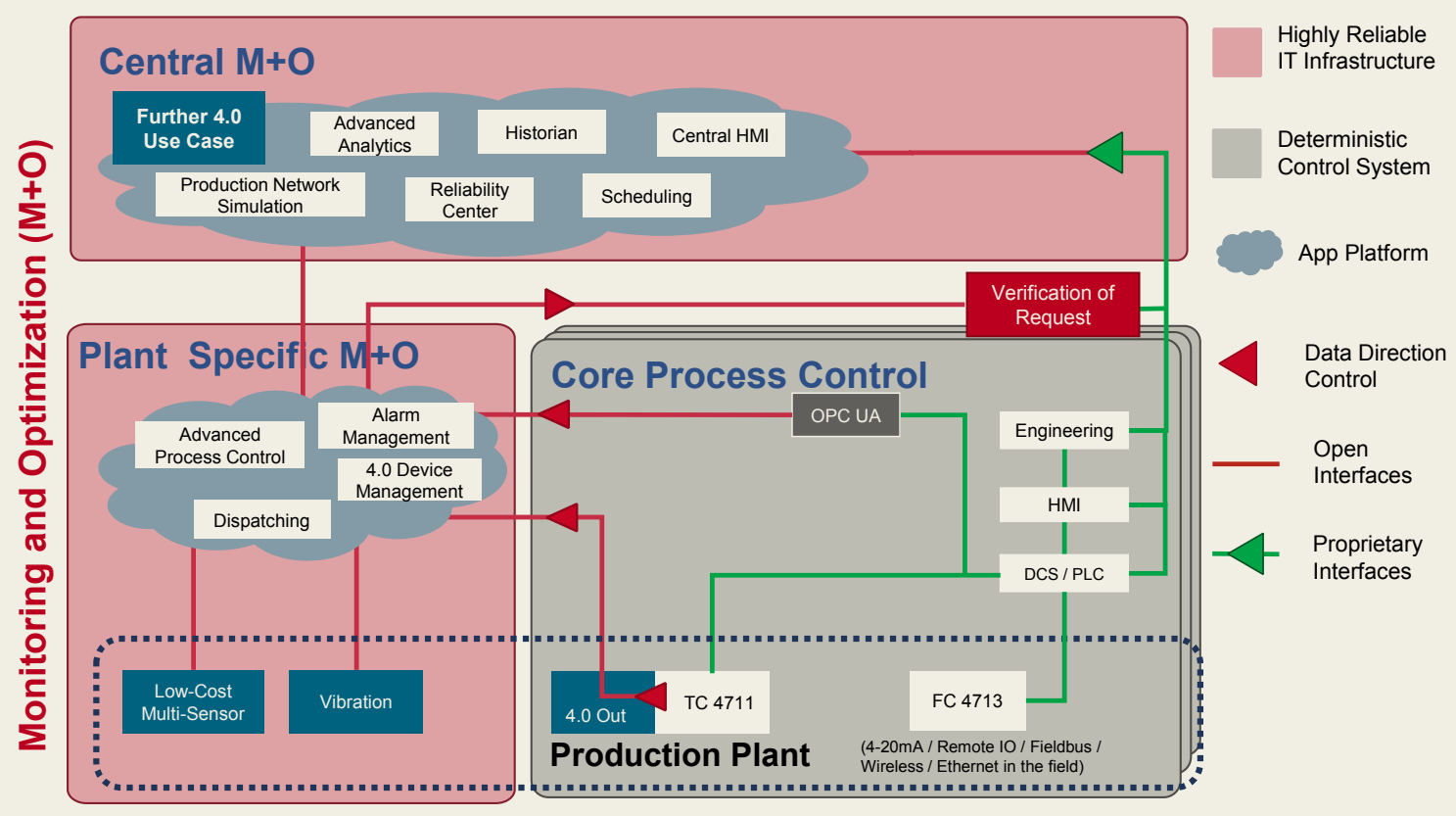

BILD 2: Struktur der Namur Open Architecture

\subsection{Offene Schnittstelle}

Die im M+O-Bereich laufenden Applikationen und Services speisen sich durch einen einfachen Zugriff auf Daten und sind durch schnelle Innovationszyklen gekennzeichnet, losgelöst von den langen Lebenszyklen der Anlagen. Offene und herstellerunabhängige Schnittstellen aus der Kernautomatisierung in den Bereich von $\mathrm{M}+\mathrm{O}$ sind hierfür eine Grundvoraussetzung. Diese Schnittstellen sorgen für eine Entkopplung zwischen den jeweiligen Bereichen sowie für einen standardisierten Übergang. Die Spezifikation solcher Schnittstellen und der zugehörigen Informationsmodelle basierend auf bestehenden Standards ist eine der Hauptaufgaben des AK 2.8.

Neben lesendem Zugriff aus der Kernautomatisierung in die $\mathrm{M}+\mathrm{O}-$ Bereiche muss ebenso eine Möglichkeit geschaffen werden, Werte aus dem rosa Bereich, beispielsweise Setpoints bei Advanced-Process-ControlAnwendungen, zurückzuschreiben. Um auch diese schreibenden Zugriffe aus dem $\mathrm{M}+\mathrm{O}-$ Bereich in die Prozessführung zu ermöglichen, werden Verificationof-Request-Funktionen benötigt. Diese Funktionen stellen einen sicheren und zuverlässigen Informationsfluss aus den $\mathrm{M}+\mathrm{O}$-Bereichen in die Kernautomatisierung dar. Die Verification of Request könnte im einfachsten Fall die Prüfung und Freigabe durch den Anlagenfahrer sein. Denkbar wären auch automatische Prüfungen, etwa auf plausible Wertebereiche oder rezeptabhängige Parameter. In diesem neuen Bereich ist noch kreative Entwicklungsarbeit erforderlich.

\subsection{Zweiter Kommunikationskanal für bestehende Feldgeräte}

Neben einer offenen Schnittstelle aus den Prozessleitsystemen in den $\mathrm{M}+\mathrm{O}-\mathrm{Bereich}$ setzt sich NOA mit der Konzeptionierung und Strukturierung eines zweiten Kommunikationskanals auseinander, um einen einfachen und flexiblen Informationsfluss aus installierten Feldgeräten zu gewährleisten und zusätzliche Information über den Prozess oder den Status des Feldgeräts zu gewinnen. Ein solcher zweiter Kommunikationskanal ist für permanente Instrumentierung nur erforderlich, wenn die Systeme der Kernautomatisierung die Daten nicht transparent und offen zur Verfügung stellen können. Gerade bei Bestandsanlagen ist dieser Ansatz interessant, da oftmals schon intelligente Geräte im Feld verbaut sind, die Daten aber weitestgehend ungenutzt bleiben.

Ein wesentlicher Punkt beim zweiten Kommunikationskanal ist die informationsschutztechnische Rückwirkungsfreiheit. Es muss sichergestellt werden, dass der zweite Kommunikationskanal keinerlei Rückkopplung auf die primäre Kommunikation und auf die eingestellten Parameter des Feldgeräts hat.

Mit heutigen Kommunikationsprotokollen, wie Hart, lässt sich eine Rückwirkung nicht ohne Weiteres vermeiden. Auch eine zusätzliche Kommunikationsschnittstelle parallel zu bestehenden Busprotokollen, wie OPC UA in den Feldgeräten, stellt als Seitenkanal ein Sicherheitsrisiko dar. Somit müssen Maßnahmen getroffen werden, um die Veränderung von Sensoreinstellungen über die zweite Schnittstelle zu unterbinden. 

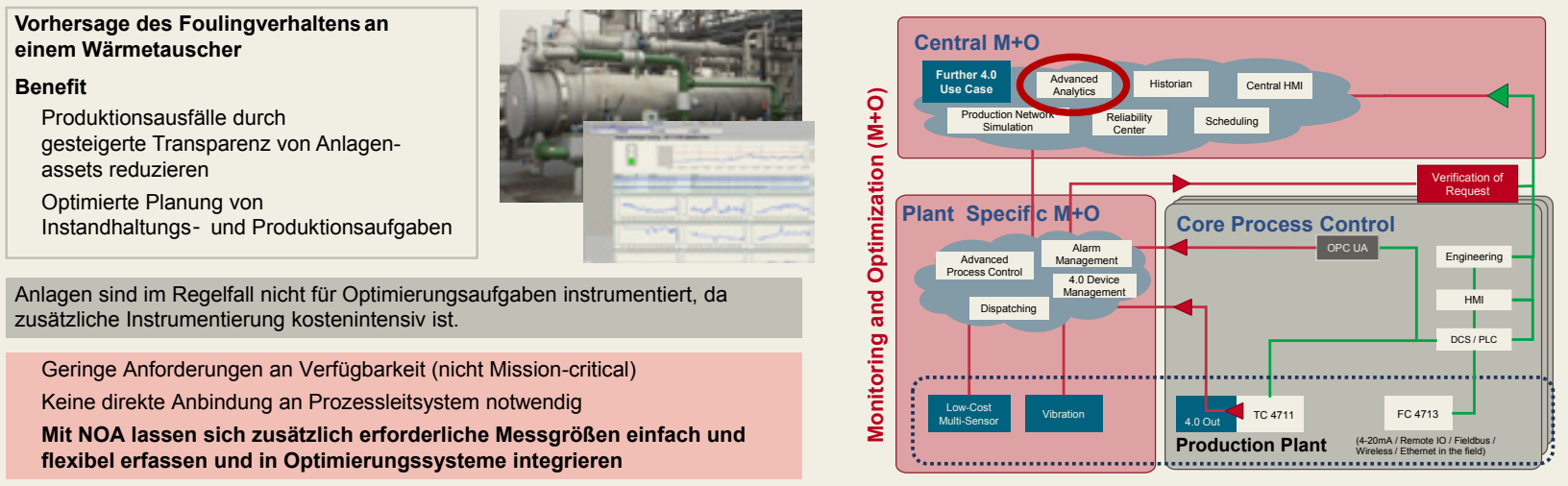

BILD 3: Beispiel Anwendungsfall Wärmetauscher

Um hier technisch tragfähige Lösungen zu erreichen, ist eine frühzeitige Mitarbeit von Feldgeräteherstellern erforderlich.

\subsection{M+O-Sensoren}

Neben bestehenden Sensoren für die Kernautomatisierung gibt NOA Raum für weitere Sensoren, um zusätzliche Information aus den Anlagen zu extrahieren. Solche Sensoren werden typischerweise bei nichtinvasiven Messprinzipien, wie Vibrationsmessungen, Verbreitung finden und können weitestgehend als IoT (Internet of Things) oder IIOT (Industrial Internet of Things) Devices verstanden werden. Wesentlich ist hier eine einfache und flexible Integration, ein aufwandreduziertes Engineering sowie ein entsprechendes Life-Cycle-Management. Über den Lebenszyklus der Anlage muss über geeignete Maßnahmen sichergestellt werden, dass $\mathrm{M}+\mathrm{O}-$ Sensoren nicht für Kernautomatisierungsaufgaben eingesetzt werden, da es sonst zu einer Vermischung der Anforderungsprofile und letztendlich der geforderten Verfügbarkeiten kommen kann. Daher sind Kernautomatisierung und $\mathrm{M}+\mathrm{O}$ strikt zu trennen.

\section{ANWENDUNGSFÄLLE}

Der Treiber von NOA ist die wirtschaftliche Realisierung von Monitoring- und Optimierungsapplikationen, um Produktionsanlagen und Prozesse weiter zu verbessern und somit kostengünstig und effizient $\mathrm{zu}$ produzieren. Nachfolgend wird an zwei Beispielen die Notwendigkeit von offenen Strukturen verdeutlicht.

Ein Beispiel für Anlagenoptimierung ist das Monitoring des Foulingverhaltens an Wärmetauschern. Unter Fouling wird die Bildung unerwünschter Beläge innerhalb von Wärmetauschern verstanden. Dieser Vorgang reduziert die Wärmeübertragungsleistung des Apparates und führt in der Regel zu reduzierter Performance, erhöhten Betriebskosten und im schlimmsten Fall zu Anlagenstillstandszeiten oder beeinträchtigt die Produktqualität.

Anlagen sind im Regelfall nicht für Optimierungsaufgaben instrumentiert, da zusätzliche Instrumentierung kostenintensiv ist. Somit lassen sich einfache Kalkulationen, wie des Wärmeübergangskoeffizienten, nicht durchführen, da Messungen nicht vorhanden sind beziehungsweise auf zusätzliche Feldgeräteinformation, wie die Temperatur an einer Durchflussmessung, nicht zugegriffen werden kann. Optimierungsaufgaben werden dadurch schon im Grundsatz erschwert oder stellen sich oftmals als unwirtschaftlich dar, da die Hürden durch eine Umsetzung in konventionellen Automatisierungsstrukturen zu hoch sind.

Dabei sind die Anforderungen bezüglich Verfügbarkeit und Echtzeitverhalten bei solchen Anwendungen eher als gering einzuordnen, und eine kostenintensive Integration in die Prozessleitebene ist oftmals nicht erforderlich.

Eine Einordnung in die NOA-Architektur zeigt Wege für eine flexible Einbindung von zusätzlichen Messgrößen. Kostenintensive PLS-Integrationen können so umgangen und Optimierungsanwendungen wirtschaftlich realisiert werden, siehe Bild 3.

Ein weiteres Beispiel findet sich im Bereich Plant Asset Management. Großmaschinen, wie Verdichter und Kompressoren, werden heute oftmals durch zusätzliche M+O-Systeme als Insellösung überwacht. Ein Transfer auf Standardequipment, wie Pumpen und Ventile, ist nur mit hohem technischen Aufwand und damit hohen Kosten möglich und hat sich bis heute in der Prozessindustrie nicht durchgesetzt. Optimierungspotenzial, wie eine großflächige Auswertung über das Betriebsverhalten von Pumpen, bleiben somit ungenutzt, da keine Assetdaten verfügbar sind. Durch die positive Preisentwicklung im Bereich von Sensoren ist jedoch zu erwarten, dass Equipment mehr und 

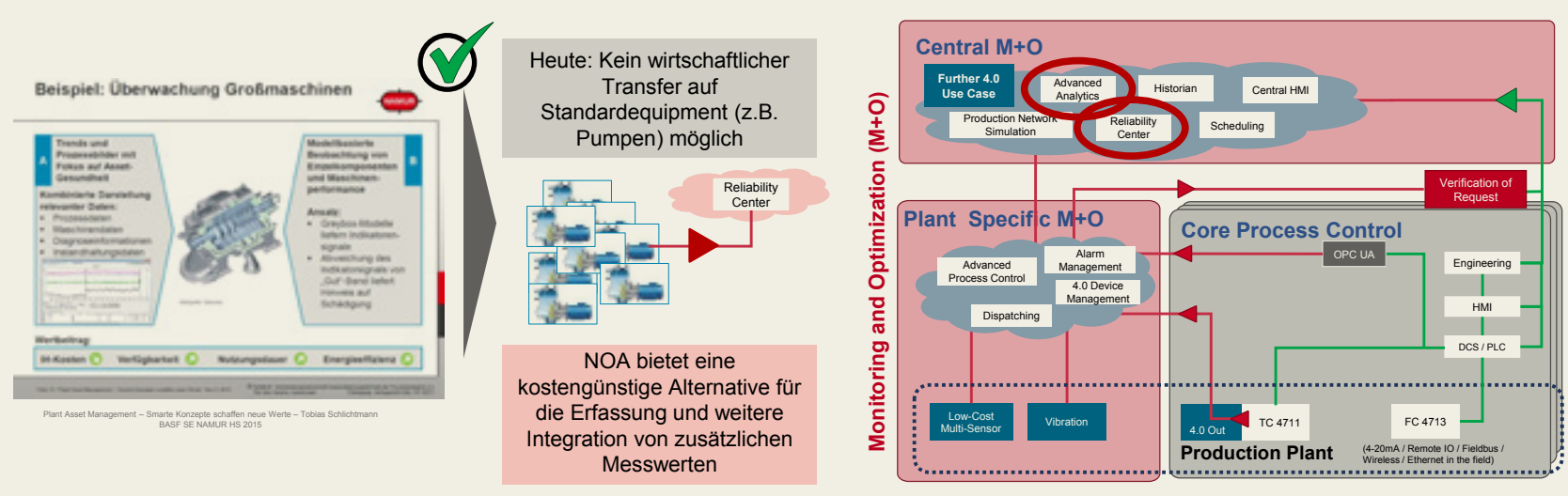

BILD 4: Beispiel Anwendungsfall Standardequipment

mehr mit Intelligenz ausgestattet wird (IoT). Somit ist es unabdingbar, die Grundarchitekturen für die kommunikationstechnische Einbindung dieser zusätzlichen Komponenten zu legen, siehe Bild 4.

Durch eine einfache und offene Architektur bietet NOA einen entsprechenden Ansatz, um Standardequipment mit entsprechenden Überwachungen auszustatten, da Messwerte von zusätzlichen Sensoren einfach und flexibel in die jeweiligen Applikationen integriert werden können, ohne die klassischen Leitsystemstrukturen mit der zusätzlichen Datenlast zu belasten.

\section{INFORMATIONSMODELL}

Um eine flexible und herstellerunabhängige Kommunikation zwischen den Elementen in der NOA-Struktur zu erreichen, wurde ein erstes Informationsmodell entwickelt, das als einheitlicher Informationsraum für Daten aus der Anlage dient, siehe Bild 5. Industrie 4.0 setzt die Verfügbarkeit von Information, beispielsweise bezüglich Fähigkeiten, Eigenschaften oder Interoperabilität von realen Komponenten, innerhalb eines Netzwerks aus intelligenten, interagierenden Teilnehmern voraus. M2M-Kommunikation erfordert die Vereinbarung von Syntax und Semantik ausgetauschter Daten bei Sender und Empfänger. Die Abbildung der Bedeutung (Semantik) ist erforderlich, um technische Systeme in die Lage zu versetzen, eigenständig Aktionen zu initiieren [7].

Das NOA-Informationsmodell definiert Typvorlagen für unterschiedliche Geräteklassen wie Druck-, Füllstand- oder Temperatur-Messgeräte. Der Informationsraum trägt im Allgemeinen Instanzen mehrerer Geräte. Wie in Bild 6 skizziert, gehört zu jeder Instanz ein Satz von charakterisierenden Parametern. Dazu zählen unter anderem Herstellername, Seriennummer, Prozesswert oder die Benutzersprache. Parameter können selbst wiederum durch Attribute charakterisiert werden. Natürlichsprachige Beschreibungen, üblicherweise in den
Handbüchern von Feldgeräten mitgeliefert, werden auf Attribute der Parameter abgebildet, auf diese Weise formalisiert und somit maschinenlesbar. Attribute können beispielsweise gemäß IEC 61360 so klassifiziert werden:

- Identifizierende Attribute (Beispiel Kennung: 0112/2///61987\#ABB271\#002, bevorzugter Name: tag name/PCE request, Versionsnummer: 002),

- Semantische Attribute (Beispiel Definition: alphanumeric character sequence uniquely identifying a measuring or control point, Quellendokument der Definition: Merkmal TAG aus IEC 61987, Formel),

- Werteattribute (Beispiel Datentyp: STRING_TYPE, Maßeinheit: entfällt, Werteliste: AKZ),

- Relationale Attribute (Beispiel Übergeordnete Klasse eines Datenelementtyps: Control Input 0112/2///61987\#ABC806\#001 oder Measured variable 0112/2///61987\#ABC407\#002).

Die beschriebene Grundstruktur des Informationsmodells, nach der ein Satz von Geräten jeweils durch Sätze von Parametern beschreibbar ist, entspringt dem Device Integration Model (DI), wie es bei FDI (IEC 62769) verwendet wird [2], das somit die Basis des NOA-Modells bildet.

Als Quelle für standardisierte Parameter dienen im NOA-Modell aktuell die 34 Core-Parameter der NE 131 [17] sowie IEC61987/eClass-Merkmale. Jede Instanz eines Parameters referenziert über eine eindeutige ID die zugehörige Typbeschreibung, siehe Bild 6. Der Aufbau der ID erfolgt für standardisierte Parameter gemäß ISO 29002-5. Zusätzlich können herstellerspezifische, nicht-standardisierte Parameter definiert und durch die Verwendung einer URI identifiziert werden. Auf diese Weise werden menschenverständliche Begriffsdefinitionen zu Metadaten der Dinge. Bild 7 verdeutlicht das Prinzip. Eine ID stellt eine Verbindung zu den in der Typbeschreibung enthaltenen Metadaten her. Diese Metadaten beschreiben ein physisches oder logisches Objekt der realen Welt 


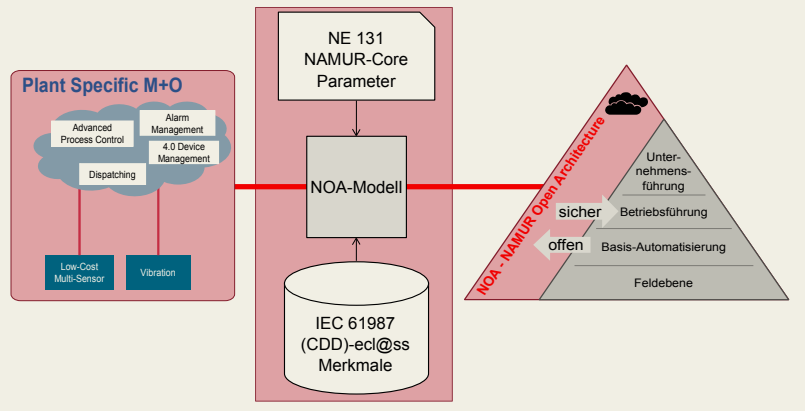

BILD 5: NOA-Modell als einheitlicher Informationsraum für Daten aus der Anlage

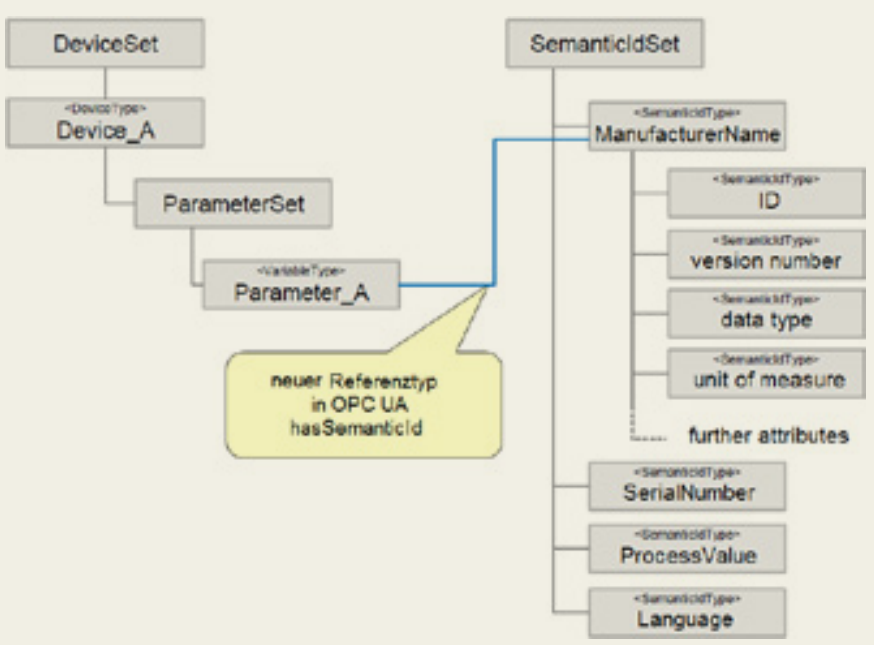

BILD 6: Grundstruktur des NOA-Informationsmodells

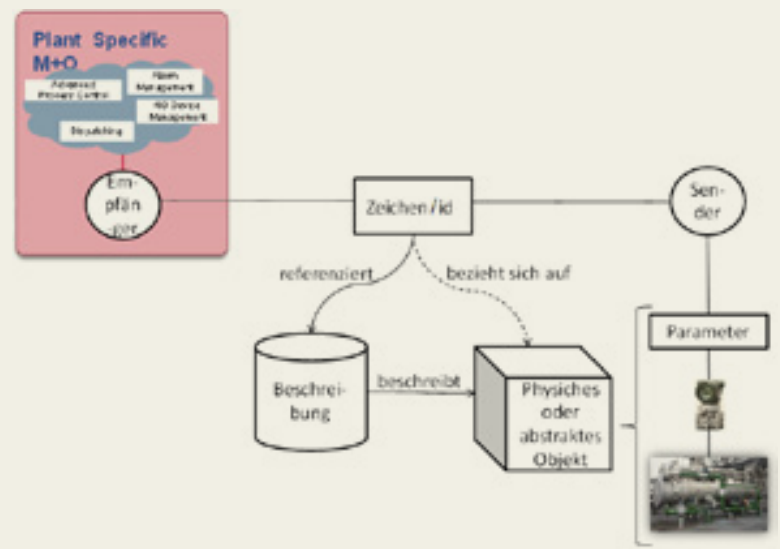

BILD 7: Einordnung des semiotischen Dreiecks in das NOA-Konzept in einer semantisch eindeutigen, maschinenlesbaren Form. Probleme natürlichsprachiger Darstellungen, wie das Vorhandensein von Homonymen, Mehrsprachigkeit und unterschiedliche Sichtweise, lassen sich so vermeiden.

Der Informationsaustausch zwischen den beteiligten Partnern umfasst mindestens die ID des Parameters sowie dessen Ausprägungsaussage [5]. Die Ausprägungsaussage umfasst den Wert des Parameters und gegebenenfalls ergänzende Attribute, wie einen Zeitstempel oder eine Angabe zur Qualität des Wertes. Auch die Charakterisierung des Aussageziels, das heißt, ob es sich bei dem Wert um eine Anforderung, eine Zusicherung oder einen Istwert handelt, ist hier möglich. Syntax-sowie Semantikbeschreibungen werden in diesem Fall nicht bei jeder Interaktion mit übertragen, sondern sind bekannt und müssen nicht zugreifbar beim Sender und Empfänger vorliegen. Andererseits sind in einem I4.0-System auch lose Kommunikationsstrukturen vertreten. In diesem Fall haben die beteiligten Instanzen zuvor keine festen Absprachen über die Semantik und gegebenenfalls auch über die Syntax auszutauschender Information getroffen. Um dennoch ein gemeinsames Verständnis der Interaktion zu gewährleisten, werden die für die jeweiligen Parameter festgelegten Identifikatoren genutzt. Lokal vorgehaltene oder systemweit zugängliche Bibliotheken ermöglichen es dann, mit Hilfe der ID Syntax- und Semantikbeschreibungen zur Laufzeit abzurufen [3].

Eine mögliche Schnittstelle im NOA Konzept ist OPC UA. Im Objektraum eines OPC-UA-Servers wären die Parameter mit ihrer ID und Wert sowie, wo erforderlich, ergänzenden Attributen einzubetten.

\section{DEMONSTRATOREN}

Die Implementierung von NOA ist als iterativer Ansatz $\mathrm{zu}$ verstehen. Um die Entwicklung agil und praxisnah zu begleiten, wurden im Rahmen der Arbeiten im Namur-AK 2.8 erste Demonstratoren erstellt. Ziel der Demonstratoren ist eine Machbarkeitsstudie, um die Umsetzbarkeit mit heutiger Technologie und verfügbaren Standards sowie die noch notwendigen Optimierungs- und Entwicklungspotenziale aufzuzeigen. Die Demonstratoren wurden im Rahmen der NamurHauptsitzung 2016 vorgestellt und werden kontinuierlich weiterentwickelt.

\subsection{Demonstrator 1 (TH Ingolstadt)}

Der Demonstrator der Technischen Hochschule Ingolstadt zeigt die Nutzung von NOA basierend auf bestehenden intelligenten Feldgeräten für Bestandsanlagen, sowie für Brownfield/Greenfield Projekte, siehe Bild 8. Intelligente Feldgeräte bieten über ihre Kommunikationsschnittstelle, zum Beispiel Profibus/Profinet, Hart, Foundation Fieldbus, Modbus, neben dem eigentlichen 


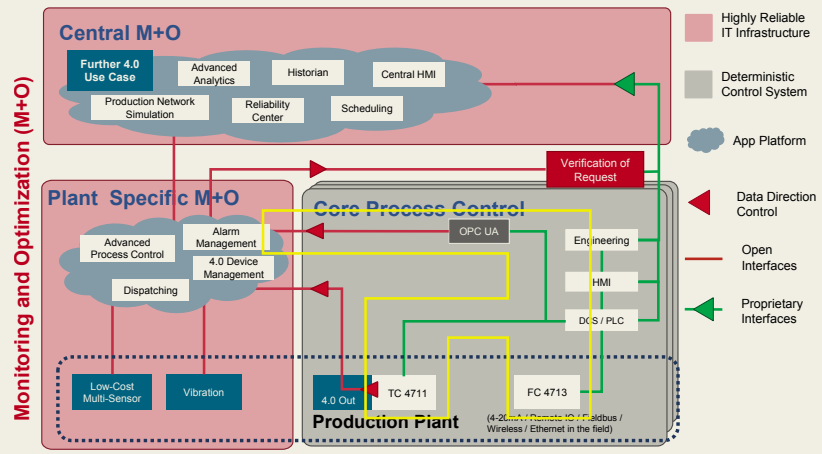

BILD 8: Einordnung des Demonstrators der TH Ingolstadt in die NOA-Architektur

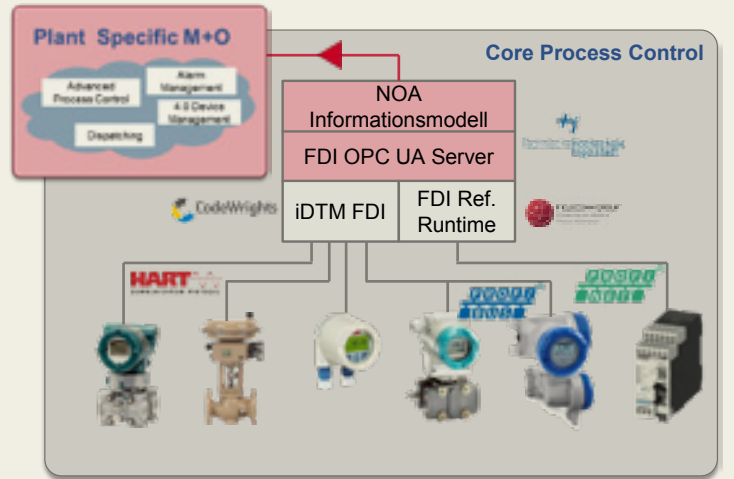

BILD 9: Aufbau des Demonstrators der TH Ingolstadt
Prozesswert bereits sehr umfassende Information über das Gerät beziehungsweise den Prozess an.

Beispiele sind aktuelle Konfigurationseinstellungen, Diagnoseinformation sowie weitere Prozesswerte, wie die Temperatur oder die Dichte des Mediums bei einem Durchflussmessumformer oder Information von Antrieben, die eine Aussage über den Zustand des Primärequipments ermöglichen. Mit dieser zusätzlichen Information lassen sich Anwendungsfälle, wie die Überwachung von Wärmetauschern oder StandardEquipment, wie Pumpen, bereits heute in Bestandsanlagen umsetzen. Der Zugang zu dieser Information ist allerdings häufig nur über proprietäre Leitsystemschnittstellen möglich. Dies führt zu einem hohen wiederkehrenden Aufwand, da leitsystemspezifische Kopplungen zu implementieren sind. Ändert sich die Leitsystemschnittstelle, zum Beispiel aufgrund eines Versionswechsels, muss die Kopplung nachgezogen werden, was wiederum Aufwand verursacht. Bietet das Leitsystem keinen Zugang oder ist dieser zu eingeschränkt, bleibt noch die Option, einen parallelen Zugang zu den Feldgeräten zu schaffen.

In jedem Fall müssen aber Teile der Topologie des Automatisierungssystems ein zweites Mal geplant und verwaltet werden. Das erzeugt nicht nur Kosten für zusätzliche Hardware, sondern schafft Aufwand für Engineering und Installation sowie Redundanzen, die bei Änderungen zu Inkonsistenzen führen können. Aus diesen Gründen wird das Potenzial der Information intelligenter Feldgeräte derzeit nur sehr begrenzt genutzt, etwa für die Gerätekonfiguration während der Inbetriebnahme. Zudem liegt die verfügbare Information in Kommunikations- beziehungsweise herstellerspezifischen Namensräumen vor, was eine einheitliche Weiterverarbeitung für die oben genannten Anwendungsfälle erschwert.
Mit Field Device Integration (FDI, IEC 62769) steht ein internationaler Standard für die Geräteintegration zur Verfügung, der bereits ein standardisiertes offenes Informationsmodell über OPC UA zur Verfügung stellt [9]. Ein FDI-OPC-UA-Server stellt ein FDI-Informationsmodell zur Verfügung und bietet damit den herstellerunabhängigen Zugang zu Feldgeräteinformation $[13,10]$. Das FDI-Informationsmodell stellt eine Weiterentwicklung des DI-Modells dar, das ebenfalls aus den FDI-Standardisierungsaktivitäten entstanden ist und die Basis für eine Reihe weiterer Informationsmodelle legt, zum Beispiel PLCopen [12]. Damit bietet ein FDI-Server eine sehr gute Basis für die Umsetzung des NOA-Informationsmodells: Der DeviceType inklusive ParameterSet und den Parameterknoten ist bereits in FDI enthalten. Für die Umsetzung des NOA-Informationsmodells ist lediglich die Verknüpfung zu den SemanticIds zu realisieren, sodass Feldgeräteparameter, die über FDI-Mechanismen zur Verfügung gestellt werden, nun zusätzlich ihre Semantik anbieten.

Der Demonstrator der TH Ingolstadt setzt diese Architektur folgendermaßen um, siehe Bild 9: Ein FDI-OPC-UA-Server bietet das in FDI spezifizierte Informationsmodell über OPC UA an. Die in der Topologie des Automatisierungssystems enthaltenen Feldgeräte finden sich im Informationsmodell als virtuelle Repräsentanten wieder. Das Informationsmodell des FDI-Servers wurde im Demonstrator erweitert, sodass nun auch die im NOA-Informationsmodell definierte Semantik verfügbar ist. Somit kann ein OPC-UA-M+OClient nun für die genannten Anwendungsfälle auf die Feldgeräte zugreifen und weitere Information über das intelligente Feldgerät, dessen Zustand sowie Prozessparameter abfragen. Da der FDI-Server die gerätespezifischen FDI-Device-Packages kennt und verarbeitet, ist er in der Lage, sowohl die NE 131-Parameter als 


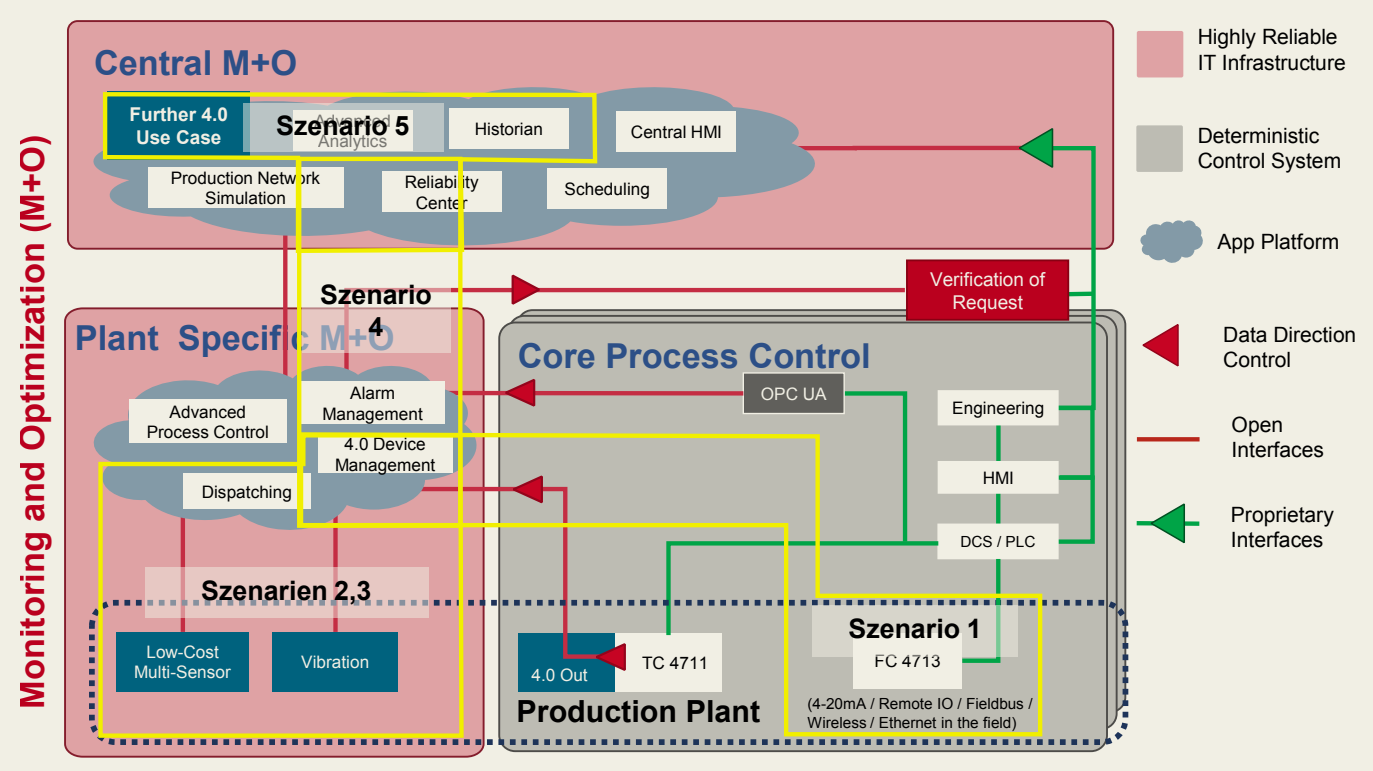

BILD 10: Verortung der Szenarien des Dresdner NOA-Demonstrators

auch geräte- und herstellerspezifische Parameter anzubieten. Für die Zuordnung der NE 131-Parameter zu den Geräteparametern im FDI-Device-Package wurden jeweils Dateien mit Mapping-Information erstellt und den FDI-Device-Packages beigestellt. Im Demonstrator wurden sechs Feldgeräte unterschiedlicher Hersteller mit drei Protokollen beispielhaft integriert:

Die Differenzdruck-Messumformer Yokogawa EJX110A (Hart) und Siemens Sitrans-P DSIII (Profibus PA) sowie der Temperaturmessumformer ABB TTH-300 (Hart) bieten neben dem Prozesswert umfangreiche Information zum aktuellen Zustand, die sich für Monitorung-Applikationen nutzen lässt. Außerdem ist der Zugriff auf die aktuelle Konfiguration und die damit verbundenen NE 131-Parameter möglich.

Der Coriolis-Massedurchfluss-Messumformer Krohne MFC400 (Profibus DP/PA) verfügt neben dem aktuellen Massendurchfluss über weitere Prozessgrößen, die für eine Optimierung relevant sein können. Dazu zählt beispielsweise die Dichte des Mediums oder aber die Erkennung von Gasblasen im Medium. Zudem lässt sich umfangreiche Information zu Gerätezustand und -konfiguration abfragen.

Stellventile zählen zu den kritischen Bestandteilen einer jeden Anlage. Demensprechend ist eine vorausschauende Instandhaltung wünschenswert. Der elektropneumatische Stellungsregler Samson 3730-6 (Hart) bietet dafür umfangreiche Information an, wie etwa den dynamischen Belastungsfaktor, der aufgrund der Belastung des Ventils langsam zunimmt. Aufgrund des Verlaufs dieses Kennwerts lässt sich eine Aussage zum Funktionsvorrat dieses Ventils treffen. Für die detaillierte Diagnose steht weitere Information im Gerät zur Verfügung, die über das NOA-Informationsmodell repräsentiert wird.

Das Motorsteuergerät Siemens Simocode Pro V (Profinet IO) verfügt über eine Vielzahl von Parametern, die eine Aussage über den Zustand des Primärequipments, wie Pumpen, ermöglichen. Dazu zählen unter anderem Wirk- und Blindleistung, Phasenverschiebung sowie das thermische Motormodell, das im Motorsteuergerät mitgerechnet wird. Diese sind über das NOA-Informationsmodell verfügbar.

Der Zugriff auf die genannten Feldgeräte und Parameter wurde im Rahmen des Demonstrators auf der Namur-Hauptsitzung erfolgreich gezeigt. Besonders hervorzuheben ist dabei, dass sich alle Feldgeräte unabhängig von Protokoll oder Hersteller im Informationsmodell einheitlich präsentieren. Das heißt, Informationsnutzer müssen sich keine Gedanken über die Spezifika des unterlagerten Kommunikationsprotokolls beziehungsweise die Topologie des Automatisierungssystems machen. Sie greifen auf die gewünschte Information im Informationsmodell zurück, und der unterlagerte FDI-Server kümmert sich um die korrekte gerätespezifische Umsetzung im jeweiligen Kommunikationsprotokoll. Für die Visualisierung der Feldgeräteinformation wurde auf das Dashboard der TU Dresden zurückgegriffen, siehe Abschnitt 5.2. Insbesondere im Zusammenspiel mit geräteintegrierten OPC-UA-Servern ist die Aggregation zu einem Gesamtmodell über einen Aggregationsserver wie in Abschnitt 5.2 und $[7,8]$ beschrieben, sinnvoll und zu empfehlen. 


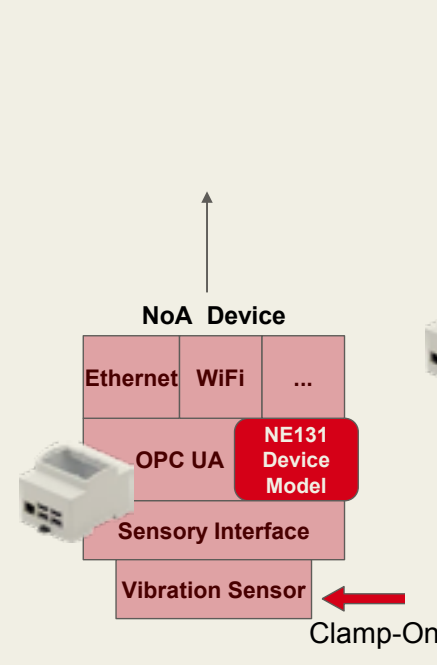

Szenario 2

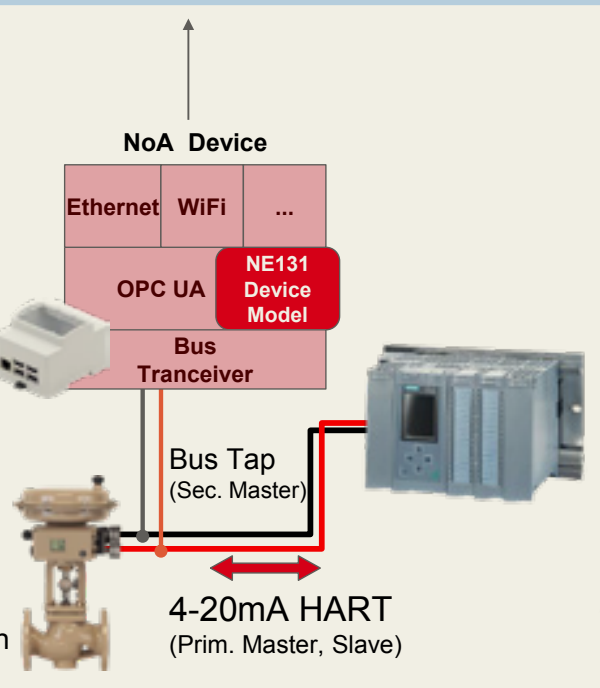

Szenario 1

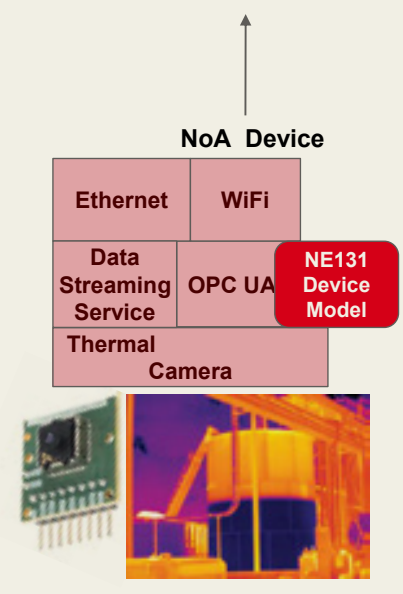

Szenario 3

BILD 11: Schematische Darstellung und Szenariozuordnung der drei datenerfassenden Geräte des Demonstrators der TU Dresden

Mit dem beschriebenen Ansatz ist es also möglich, die umfangreiche Information intelligenter Feldgeräte standardisiert und offen zu nutzen. Ein Arbeitspunkt ist die Zuordnung der Geräteparameter im FDI-Package zu den NE 131-Parametern. Hier wurde bereits die Abstimmung mit der FieldComm Group und der Profibus-Nutzerorganisation angestoßen, um einen einheitlichen Weg zu definieren, sodass der im Demonstrator gegangene Umweg über Mappings vermieden wird.

\subsection{Demonstrator 2 (TU Dresden)}

Der Demonstrator der Professur für Prozessleittechnik der Technischen Universität Dresden untersucht die Aspekte vertikale Integration und Informationsnutzung. Im Fokus steht die effiziente Einbindung der durch NOA bereitgestellten Information in semantische Netze. Diese erhöht die Fähigkeit verfahrenstechnischer Anlagen zur schnellen, weitgehend automatisierbaren Anpassung an volatile Produktionseinflüsse durch Plug-and-produce oder Measurement-ondemand, wenn Aspekte der Informationssicherheit und der Gebrauchstauglichkeit zu berücksichtigen sind [6].

Ausgangspunkt des Demonstrators ist die Bestandsautomatisierung einer fiktiven prozesstechnischen Anlage. Repräsentiert wird diese durch eine Steuerung (Siemens S1500) sowie einen über 4-20mA und Hart angebundenen pneumatischen Stellantrieb (Samson). Auf dieser Hardwarebasis werden die in Bild 10 dargestellten fünf Szenarien in je einem Teildemonstrator umgesetzt.

\subsubsection{Hard- und Software}

Drei Geräte des Demonstrators sind für die Erfassung von Information in Anlagennähe verantwortlich. Der Entwurf der Geräte orientiert sich dabei an der Architektur eines Mehrwertadapters [14]. Eine anwendungsspezifische Schnittstelle erfasst die Daten aus der jeweiligen Informationsquelle (Sensor, Aktor, Feldbusgerät). Die so gesammelten Daten werden von einem OPCUA-Server zugänglich gemacht, der diese durch das NE 131-Informationsmodell semantisch annotiert. Die gegebenen Geräte versenden periodisch UDP-Broadcasts, sodass hinzugefügte oder entfernte OPC-UAInformationsquellen lokalisiert werden können. Ein viertes Gerät detektiert diese Quellen und aggregiert deren OPC-UA-Information automatisch. Jedes der im Demonstrator eingesetzten Geräte wird von einem Raspberry Pi betrieben, der per WLAN oder Ethernet Zugriff zum OPC-UA-Server (open-source-Stack open62541 [18]) gewährt. Die Anbindung an die industrielle Kommunikation erfolgt über Schnittstellen, die von der TU Dresden für den NOA-Demonstrator entwickelt wurden. Schlussendlich ist die IP-Infrastruktur, also WLAN Access Point und Ethernet-Switch, Teil der Demonstrationsplattform.

\subsubsection{Szenario 1: Semantische Integration von Altgeräten}

In diesem Szenario, siehe Bild 11, werden Daten eines bereits in der Anlage verbauten Feldgeräts angezapft. NOA stellt den relevanten Ausschnitt des Datenhaushalts semantisch annotiert zur Verfügung. Die Funktion 


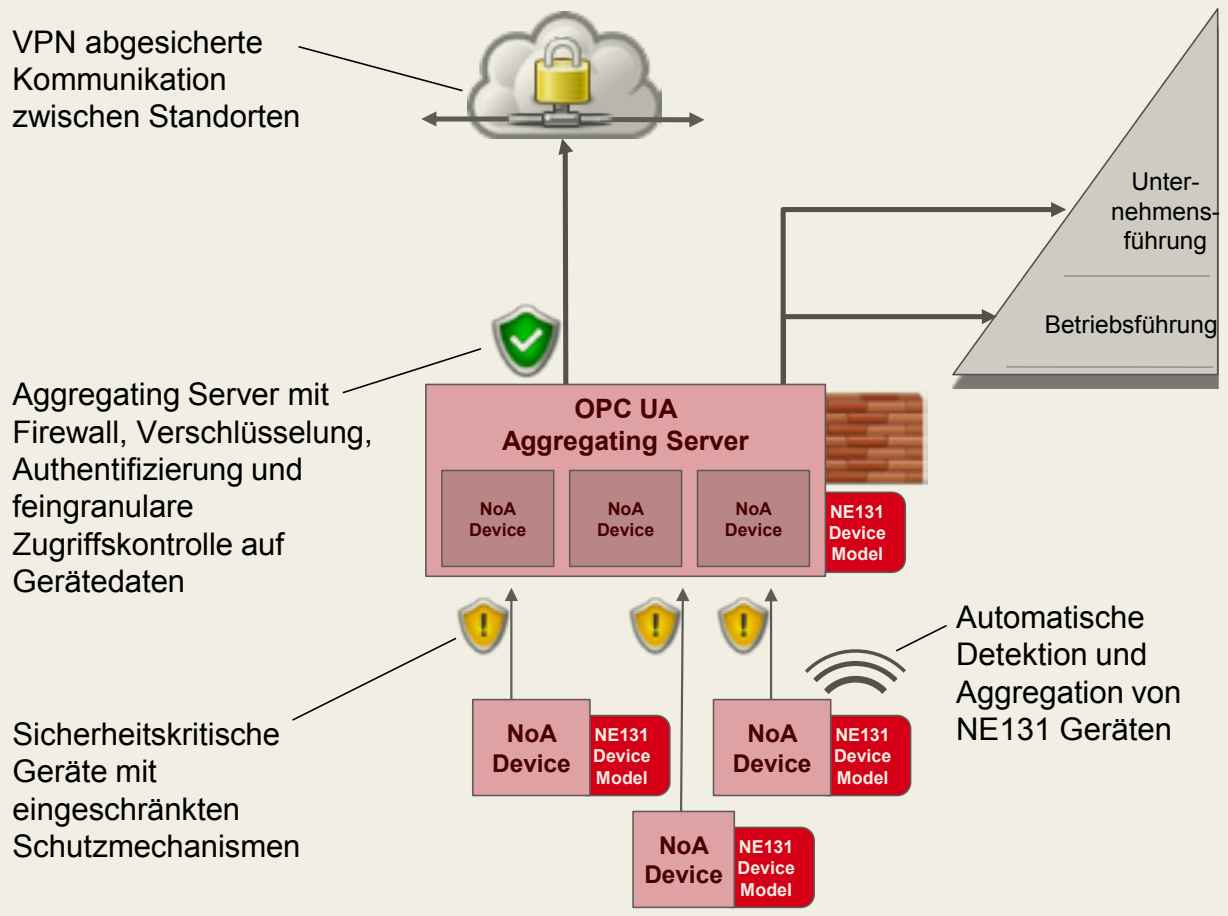

BILD 12: Darstellung der Rolle des Aggregating-Servers in einer NOA-Architektur

des Feldgeräts als Teil der Prozessführung soll dabei nicht beeinflusst werden. Wesentliches Alleinstellungsmerkmal dieser Teildemonstration ist, dass für die Anbindung des für den NOA-Ansatz datenerfassenden Geräts weder neue Sensoren noch Feldbusmaster-Bausteine in den Bestand eingebracht werden. Dies funktioniert immer dann, wenn bestehende Feldbusse Secondary/Multi-Master Modi beziehungsweise frei allozierbare Zeitscheiben zur asynchronen Kommunikation bereitstellen. Für das Auslesen der Gerätedaten sind drei Modi denkbar, die jeweils unterschiedliche Konsequenzen für das Security-bydesign haben:

- Passiv lesend (mithören der Kommunikation zwischen Steuerung und Feldgerät). Die erfassbaren Daten sind gleichzeitig auf die Informationsinhalte der erfassten Kommunikation beschränkt. Lediglich Authentizität und Vertraulichkeit sind kompromitierbar.

- Aktiv lesend (versenden von Lesebefehlen an das Feldgerät). Durch die Beeinflussung der Master/ Slave-Kommunikation kann die Verfügbarkeit kompromitiert, aber ebenso beliebige Daten des Feldgeräts erfasst werden.

- Aktiv lesend/schreibend (zusätzliche Schreibbefehle). Alle Angriffsmöglichkeiten werden möglich und die Ausgestaltung eines effizienten Zonen-Übergangs wird erforderlich.
Der implementierte Teildemonstrator für dieses erste Szenario greift als Hart-Secondary-Master aktiv lesend auf den Bus zu. Feldgeräte werden über Hart identifiziert und Daten wie Hersteller, Gerätetyp und (periodisch) der primäre Prozesswert ausgelesen. Hart stellt hier ein ideales Protokoll für die Demonstration der NOA-Fähigkeiten dar, da in entsprechenden Feldgeräten oft mehr als 100 Datenwerte Auskunft über Prozess und Lebenszyklus bieten. In der Praxis wird diese Informationsfülle selten genutzt und Hart lediglich als Konfigurationsschnittstelle eingesetzt. Die für Diagnose und Optimierung wertvolle Information wird durch NOA für die algorithmische Weiterverarbeitung wieder zugänglich gemacht.

\subsubsection{Szenarien 2 und 3: Effiziente Integration temporärer Sensorik}

Die Szenarien 2 und 3, siehe Bild 11, agieren unter der Prämisse, dass Prozessabschnitte der Bestandsanlage für fortgeschrittene Prozessoptimierungsmethoden zusätzliche Sensorik benötigen. Die Szenarien unterscheiden sich in Hinsicht auf die dafür notwendige Bandbreite. In Szenario 2 wird lediglich ein Vibrationssensor eingebracht, dessen Kommunikationsanforderungen auch durch konventionelle Feldbusse abgedeckt werden könnten. Szenario 3 überwacht einen Prozessabschnitt mittels einer Thermografiekamera 
(Video-Datenstrom), deren Bandbreitenanforderung nicht rückwirkungsfrei über etablierte Feldbustechnologien bereitgestellt werden kann. In beiden Szenarien soll die laufende Anlage nicht durch das Einbringen neuer Sensorik modifiziert und die Geräte minimalinvasiv (Clamp-On) an die Anlage angeschlossen werden.

Ein anwendungsnahes Beispiel für Szenario 2 ist eine Pumpe, an der Kavitation durch einen zusätzlichen Vibrationssensor festgestellt werden soll. In Szenario 3 könnte die gleiche Pumpe in ihrer Gesamtheit auf Hitzeentwicklungen an Antrieb oder mechanischen Komponenten überwacht werden, an denen die Montage zusätzlicher Sensorik nicht möglich ist. Auch andere breitbandige Datenströme wie Audiodaten für die akustische Diagnose von Feldgeräten wären potenzielle Anwendungsfelder für das Szenario.

Der Teildemonstrator 2 basiert auf einem handelsüblichen Beschleunigungssensorbaustein von Analog Devices. Der Raspberry Pi hinterlegt die ermittelte Beschleunigung in der OPC-UA-Process-Value-Variable. Teildemonstrator 3 verwendet ein FLIR Lepton Thermografiemodul, um einen http-Video-Feed bereitzustellen. OPC UA bildet die URL, Eigenschaften des Videostreams und des verwendeten Thermografiesystems ab.

\subsubsection{Szenarien 4 und 5: Vertikale Integration}

Szenario 4 des Demonstrators ist der NOA-Aggregating-Server. Es handelt sich um einen OPC-UAServer, der wie die bereits vorgestellten Geräte das NE 131-Gerätemodell beherrscht, siehe Bild 12. Der Server sucht kontinuierlich nach den UDP-Broadcasts von datenerfassenden Geräten. Wird ein neues Gerät detektiert, spiegelt der Aggregating-Server den Informationshaushalt des Geräts in einem neuen OPC-UAObjekt. Die Spiegelung verwendet OPC-UA-Subscriptions, um Werte kontinuierlich zu aktualisieren. Schreiboperationen auf solchen aggregierten Variablen oder Methoden werden an das Quellgerät weitergeleitet. Ein Aggregating-Server enthält damit die Information aller anlagennahen Geräte des NOA-Ansatzes, die sich im Netzwerk als solche zu erkennen geben. Sendet ein Gerät über eine längere (definierbare) Zeit keine UDPBroadcasts, wird der Informationsgehalt des Geräts wieder aus dem Aggregator entfernt.

Der Aggregating-Server erfüllt zwei maßgebende Rollen für den NOA-Ansatz. Primär setzt der Server das Plug-and-play-Konzept um, indem er als zentraler Ansteuerpunkt für alle OPC-UA-Information dient. Datenerfassende Geräte werden automatisch detektiert und dargestellt. Inbetriebnahmekonfigurationen an den datenerfassenden Geräten entfallen. In seiner zweiten Rolle entkoppelt ein Aggregating-Server kommunikationstechnisch Informationssenken von den Quellen (Proxy). Gängige Sicherheitsmechanismen können an zentraler Stelle implementiert werden.
Zukünftig könnten in diesem System Zugriffsrechte auf bestimmte Daten vergeben, Verschlüsselungsund Authentifizierungsmechanismen eingesetzt und eine Firewall umgesetzt werden. Das Verfahren ist ausschlaggebend für den Einsatz von NOA in realen Netzwerkumgebungen, da ein ungeregelter Seitenkanal bis zu der Information der Anlage eine Vielzahl ITsicherheitstechnischer Konsequenzen nach sich zieht.

Im abschließenden, fünften Szenario wird die flexible Nutzung des Informationshaushaltes durch das an der TU Dresden entwickelte NOA-Dashboard gezeigt, siehe Bild 13. Es handelt sich um ein NodeJS-basiertes WebFrontend, dem eine REST-Schnittstelle den lesenden und schreibenden Zugriff auf den OPC-UA-Informationshaushalt des Aggregating-Servers und damit auf alle im Netz aktiven Geräte ermöglicht. Das NOA-Dashboard, im Demonstrator über den Browser eines generischen Tablets zugänglich, verdeutlicht Szenarien wie die mobile Wartung und Instandsetzung. Die Schnittstelle zum Nutzer agiert vollständig konfigurationsfrei: Wird einer der Teildemonstratoren aus Szenarien 1 bis 3 eingeschaltet, erscheint dieser nach kurzer Zeit gespiegelt im Aggregating-Server (Szenario 4) und in der Übersicht des Webinterfaces (Szenario 5). Geräteparameter können auf einer Detailansicht eingesehen und - sofern gestattet - modifiziert werden. Eine Trendlinie aller Prozesswerte und der Videostream der Thermografiekamera sind ebenfalls zugänglich.

\subsection{Demonstrator 3}

\section{(Otto-von-Guericke-Universität Magdeburg )}

Der Demonstrator stellt eine prototypische Implementierung eines Automation-Gateways (AG) dar. Dieses bietet eine sichere Ankopplung von Feldgeräten aus dem Bereich der Kernautomatisierung an Cloud-Applikationen aus dem $\mathrm{M}+\mathrm{O}-\mathrm{Bereich}$ über eine offene OPCUA-Schnittstelle, siehe Bild 14. Ein solches Gateway kann in der Praxis durch eine Remote-IO-Baugruppe realisiert werden, die mit der benötigten GatewayFunktionalität ausgestattet wird.

Für Anwendungen des $\mathrm{M}+\mathrm{O}$ kann das AutomationGateway Sekundärwerte bestehender Geräte, zum Beispiel Temperaturwert von Druckmessgeräten, oder zusätzliche Geräte anbinden, beispielsweise über Hart oder Profibus PA. Auf diese Weise lassen sich zusätzlich Messgrößen ohne größeren Aufwand für beispielsweise Advanced Process Control nutzen oder in andere Applikationen des $\mathrm{M}+\mathrm{O}$ einbinden, siehe Bild 15.

Die Implementierung des AG basiert aktuell auf einer SPS von Wago mit E/A-Modulen. Dies dient als Prototyp für die besagte Remote-I/O. Hierüber sind ein Hartfähiges Differenzdruckmessgerät und ein Hart-fähiger Temperaturtransmitter von Yokogawa angeschlossen. Es erfolgt eine Abbildung von Gerätezugriffen der SPS (Hart Universal und Common Practice Commands) auf einen SPS-internen OPC-UA-Server mit herstellerspezifischem 

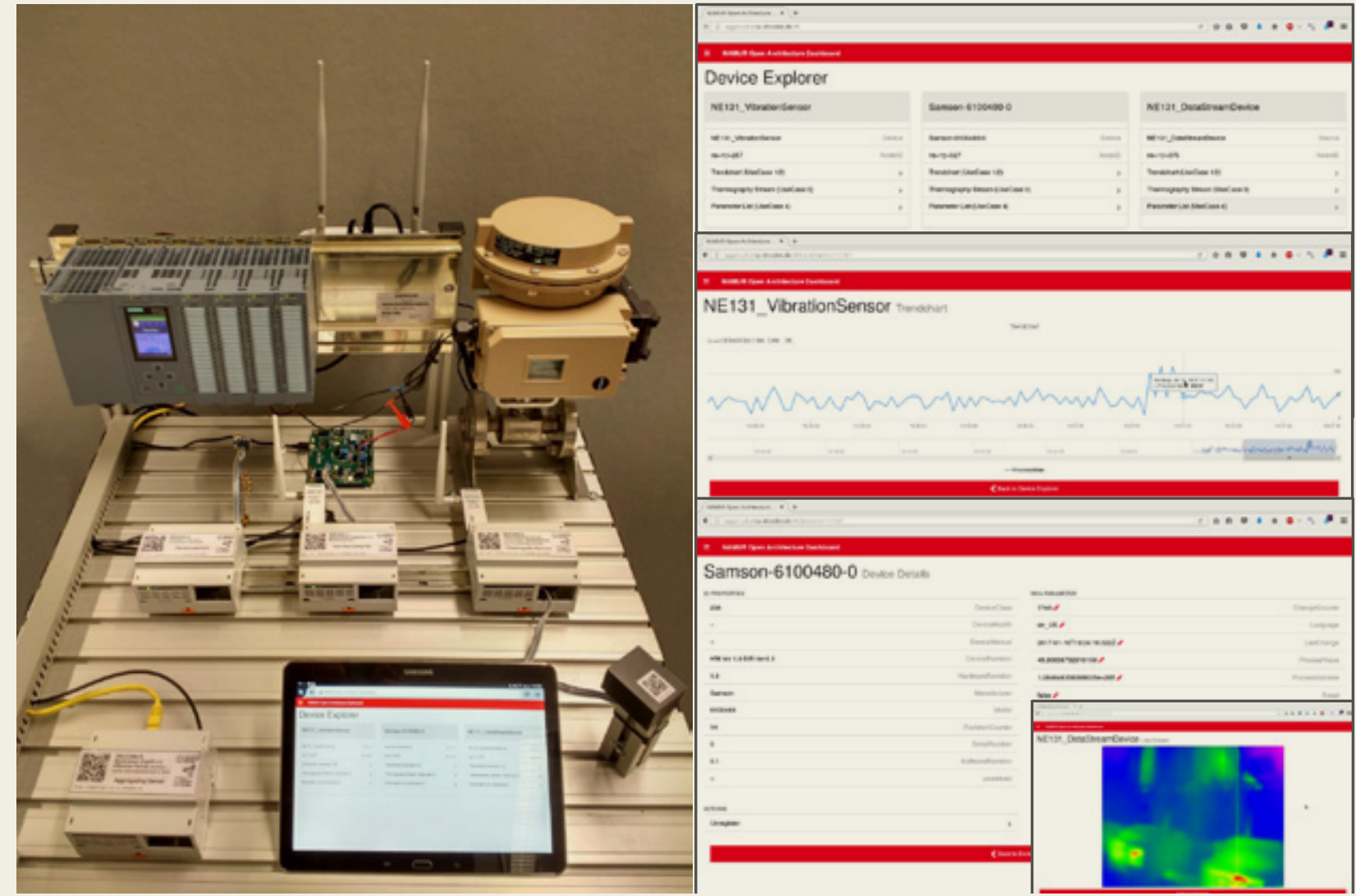

BILD 13: NOA-Demonstrationsplattform der TU Dresden (links) und Ansichten des NOA-Dashboards (von oben: Geräteübersicht, Trendlinien des Prozesswerts, Gerätedetails und Video-Stream der Thermografiekamera)

Informationsmodell. Ein weiterer, separater OPC-UAServer mit integriertem NOA-Informationsmodell greift auf den SPS-internen Server zu und führt ein Mapping auf das NOA-Modell durch. Zukünftig soll dieser separate Server direkt in dem Gateway implementiert sein.

Der Zugriff auf den OPC-UA-Server mit dem NOAModell kann über ein zusätzliches Ethernet/IP-Segment oder zum Beispiel über Profinet geschehen. Das enthaltene NOA-Informationsmodell stellt für jedes Gerät standardisierte, über eine ID identifizierbare Geräteparameter aus der NE 131 und IEC61987/eClass bereit. Parameter, die nicht standardisiert sind, können beispielsweise über herstellerspezifische URI identifizierbar gemacht werden. Durch die Verwendung von ID (ISO-29002-5, URI) sind die Daten semantisch eindeutig. Das schafft die Voraussetzung für die in der Industrie 4.0 geforderte Interoperabilität technischer Systeme.

Der Demonstrator zeigt, wie durch Nutzung eines Automation-Gateways Daten Hart-fähiger Feldgeräte einfach und flexibel über standardisierte Protokolle
(OPC-UA) übergeordneten Applikationen verfügbar gemacht werden können. Zukünftig ist der Ansatz auf weitere Kommunikationsprotokolle zu übertragen (zum Beispiel Profibus PA, Profinet), sodass ein protokollunabhängiger Zugriff über das NOA-Informationsmodell möglich ist. Derartige Automation-Gateways müssten von Herstellern angeboten werden. Entscheidend für deren Anwendbarkeit ist die Umsetzung des Verification of Request als auch weiterer Schutzmechanismen, wie die Verschlüsselung der Kommunikation mit der Cloud oder die Nutzung signierter Firmware.

\subsection{Demonstrator 4 (RWTH Aachen)}

In der Prozessmesstechnik werden heute überwiegend Feldgeräte eingesetzt, die mit erheblichen Speicher- und Rechenressourcen ausgestattet sind. Diese Ressourcen werden genutzt, um vielfältige Messfunktionen, Messwertvorverarbeitungsfunktionen und Selbstdiagnosefunktionen im Gerät auszuführen. 


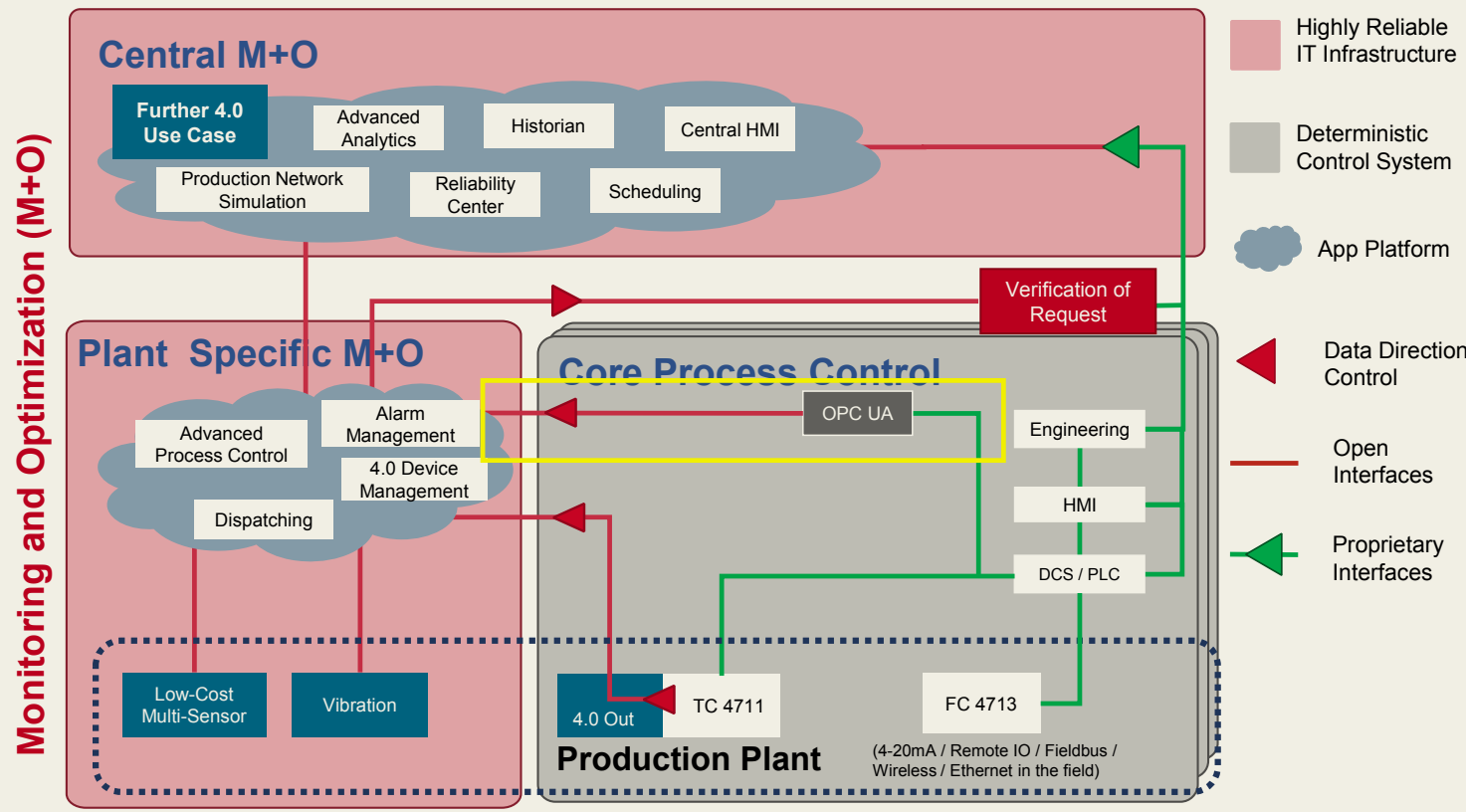

BILD 14: Einordung des Demonstrators der Universität Magdeburg in die NOA-Architektur

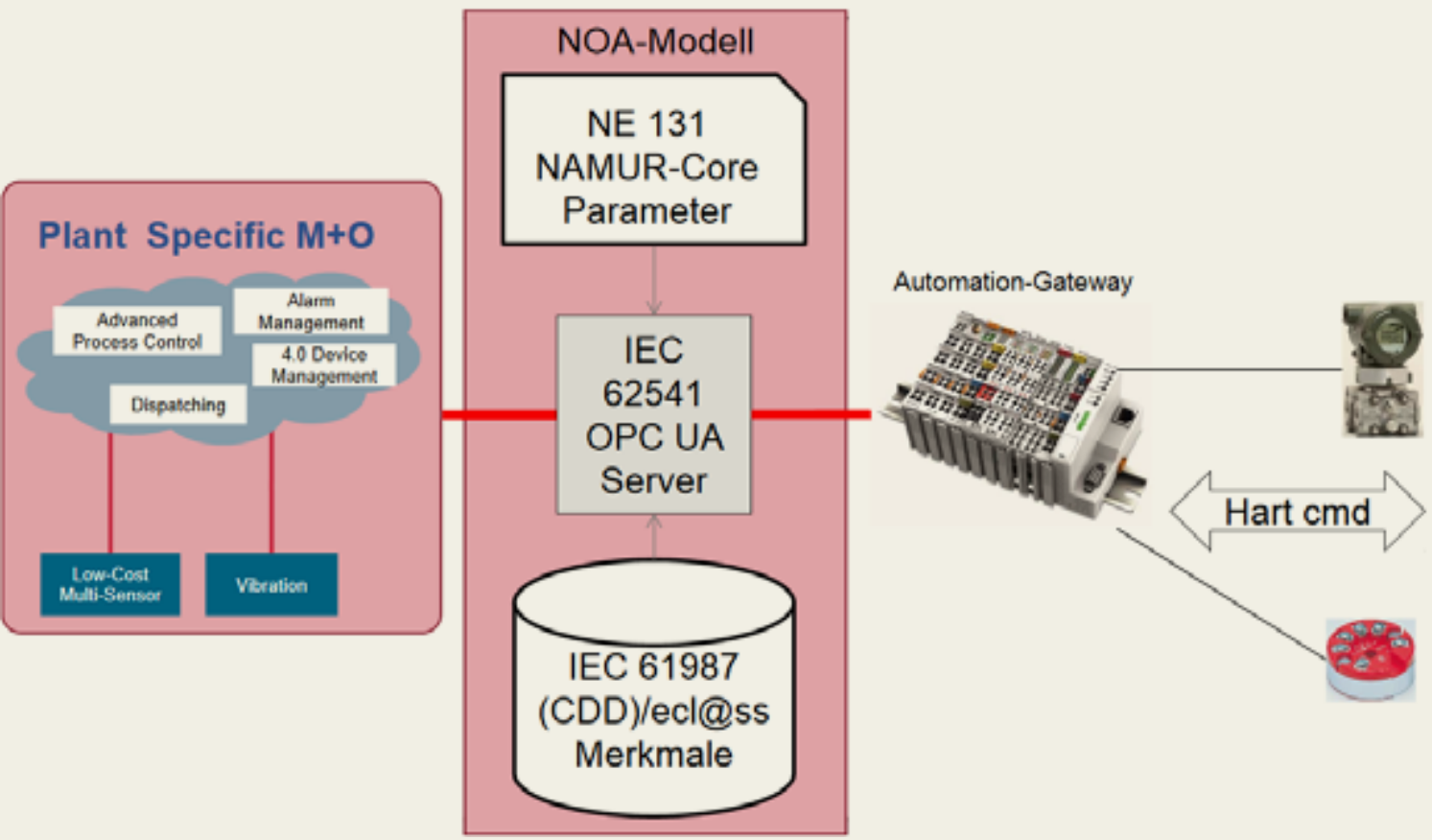

BILD 15: Anbindung an Cloud-Applikationen durch Automation-Gateway

Damit entsteht im Gerät umfangreiche Information, die auch für übergeordnete M+O-Dienste von Interesse wäre. Die klassische 4-20mA-Technologie bietet jedoch keine effiziente Möglichkeit, um an diese Information heranzukommen. Sämtliche an die übergeordneten M+O-Dienste zu übermittelnden Daten müssten über das 4-20mA-Hart-Protokoll übertragen und durch die Automatisierungssysteme verwaltet und durchgeroutet 


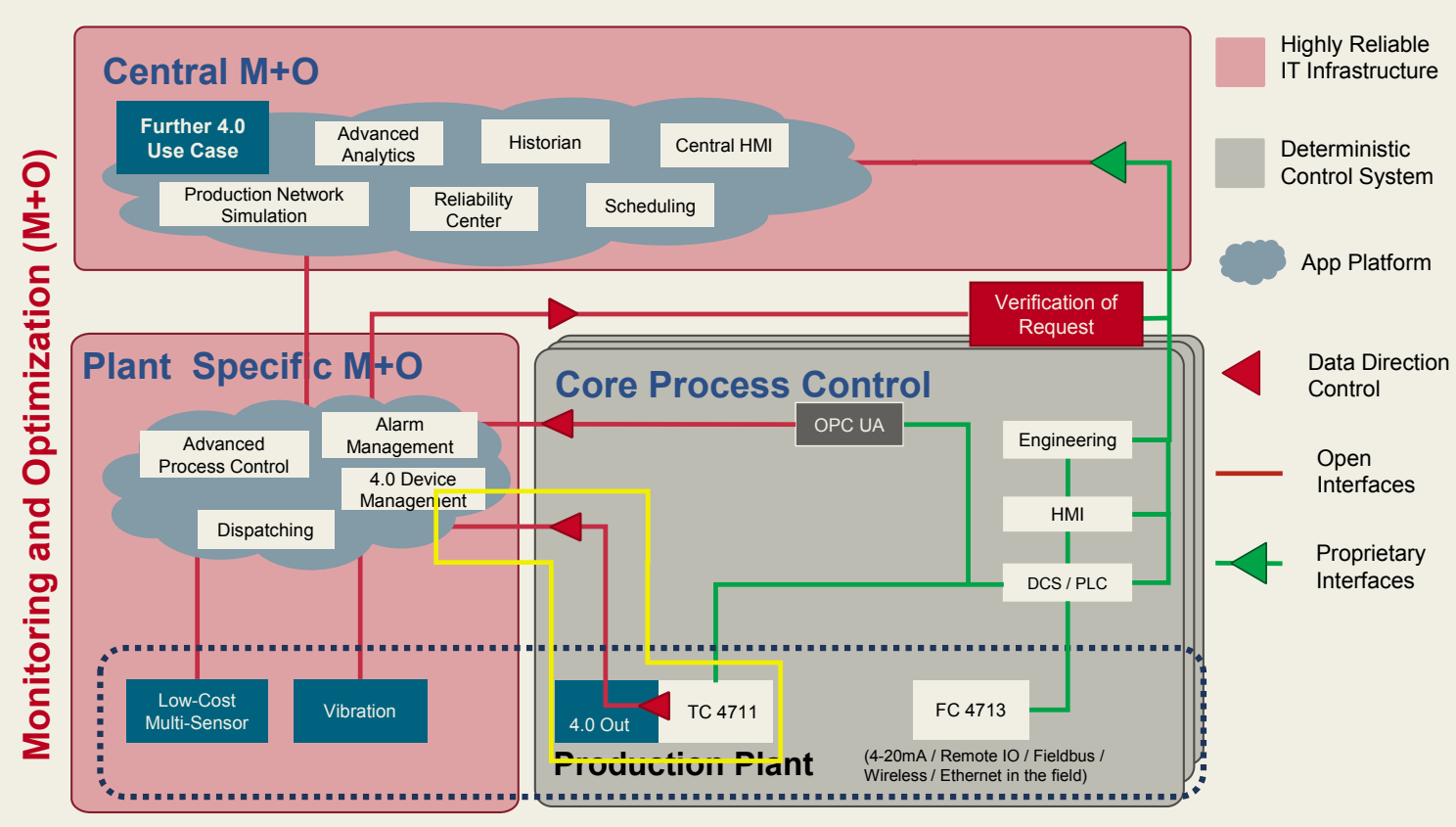

BILD 16: Einordung des RWTH-Demonstrators in die NOA-Architektur

werden. Dies ist technisch und organisatorisch aufwendig und ineffizient. Ein Anwendungsszenario von NOA ist die Implementierung eines zweiten Kommunikationskanals zur direkten parallelen Übertragung dieser Information vom Feldgerät an die M+O-Dienste. Der von der RWTH Aachen und Krohne entwickelte Demonstrator 4 dient zur Darstelung dieses Szenarios.

Die Basis des Demonstrators bildet ein Corriolisdurchflussmesser von Krohne, siehe Bild 16. Dieser ist klassisch über ein 4-20mA-Signal (mit Hart) an das Leitsystem angebunden (grüne Linien im Core Process Control). Für die Demonstration wurde von Krohne mit einem Ergänzungsmodul eine zusätzliche Recheneinheit im Feldgerät implementiert und an den internen Bus des Messumformers angeschlossen (rote Linie von I4.0 Out). Auf dem Ergänzungsmodul befindet sich ein I4.0-Device-Manager, an den jede wichtige Information des Messumformers übertragen wird. Die Datenübertragung basiert auf einem rein lesenden Zugriff, um die Informationsdiode intrinsisch zu realisieren. Das I4.0-Device-Management verwaltet die empfangenen Daten in einem OPC-UA-Server. Über den zweiten Kommunikationskanal kann auf diese Daten mit OPC-UA-Diensten offen zugegriffen werden. Die Semantik des Metamodells entspricht dem FDI-Feldgerätestandard.

In der prototypischen Implementierung bildet ein Pi Zero die zusätzliche Recheneinheit auf dem Ergänzungsmodul. Der Pi Zero besitzt einen WiFi
Access Point zur Realisierung des zweiten Kommunikationskanals mit WLAN, siehe Bild 17. Auf Grund der geringen Baugröße des Pi Zero konnte die gesamte Erweiterung im Gehäuse des Messumwandlers untergebracht werden. So bleibt die IP-Schutzklassifikation erhalten.

Über die OPC-UA-Schnittstelle kann sich die im Netz befindliche Verwaltungsschale effizient mit aktueller Information und Meldungen versorgen. Die Verwaltungsschale bildet für alle übergeordneten $\mathrm{M}+\mathrm{O}-$ Dienste die zentrale Anlaufstelle für alle das Feldgerät betreffende Information. Sie verwaltet die Historie und kennt den aktuellen Zustand des Feldgeräts. Einmal übernommene Daten stehen auch in Offlinephasen des Feldgeräts zur Verfügung. Der Demonstrator zeigt, dass dieses Konzept für Vierleitergeräte technologisch umsetzbar ist.

Zweileitergeräte können nicht mit dem oben beschriebenen Verfahren in die NOA-Architektur integriert werden, da über das 4-20mA-Signal nicht genug Leistung bereitgestellt wird. Um diese trotzdem integrieren zu können, wurde ergänzend ein Konzept zur Anbindung über Bluetooth Low Energy entwickelt, siehe Bild 18. Ein bereits in der Nähe vorhandenes Vierleitergrät dient hierbei als Hub, um die Daten zu sammeln und über OPC UA zur Verfügung zu stellen.

Mit der reinen Bereitstellung der Daten ist das Potenzial des zusätzlichen I4.0-Device-Managers noch nicht ausgeschöpft. Ein nächster Schritt wäre die Implementierung einer App-Struktur zur lokalen Ausführung 


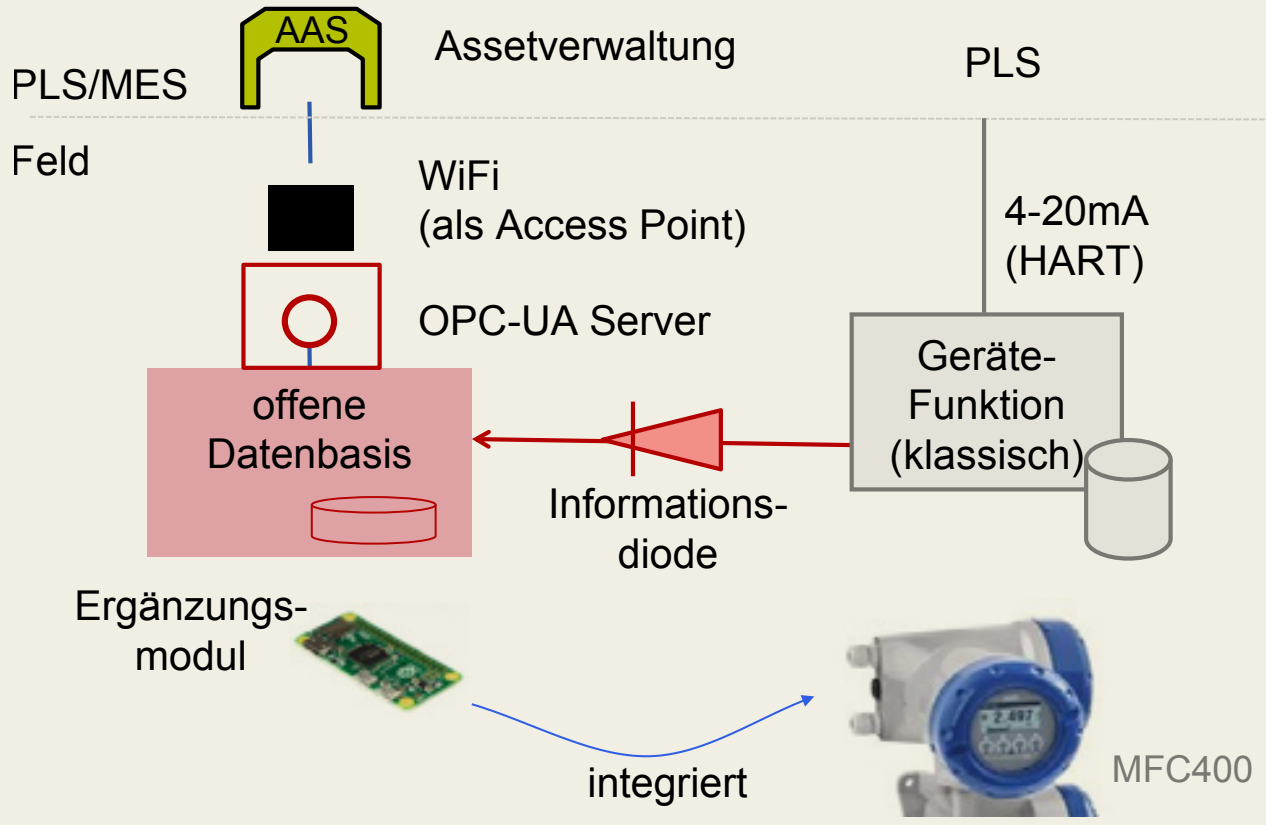

BILD 17: Erweiterung eine Feldgeräts um den zweiten Kanal

von Diagnose- und Statistikfunktionen. Ein weiterer Anwendungsfall ist die lokale Speicherung von wichtigen Lebenszyklusdaten auf dem Gerät.

\section{ZUSAMMENFASSUNG UND AUSBLICK}

Die Namur Open Architecture bietet einen konzeptionellen Ansatz für eine offene Struktur in der Prozessautomatisierung, sowohl für Bestands- als auch für Neuanlagen. Der Ansatz ist additiv zu bestehenden Strukturen und stellt eine Grundlage für die Implementierung von neuen innovativen Anwendungen im Rahmen von Industrie 4.0 dar.

Folgende Leitlinien und Kernanforderungen sind dabei zu berücksichtigen:

- Keine Kompromisse bei Anlagenverfügbarkeit und Anlagensicherheit;

- Offene Systemschnittstellen zwischen Process Control und Monitoring \& Optimization;

- Ansatz muss sowohl für neue Produktionsanlagen als auch für bestehende Produktionsanlagen nutzbar sein;

- Agile Umsetzung basierend auf bestehenden Standards;

- Automation Security ist integraler Bestandteil (Security by Design);

- Anwendbarkeit, Reduktion der Komplexität und Wirtschaftlichkeit sind wesentliche Erfolgsfaktoren. Der Schwerpunkt der weiteren Entwicklung liegt auf der Konzeptionierung der Informationsmodelle, der jeweiligen Schnittstellen und des zweiten Kommunikationskanals. Detaillierte Security-Betrachtungen, um eine sichere Kommunikation zwischen $\mathrm{M}+\mathrm{O}$ und Core Process Control sicherzustellen und Fragen zum Life Cycle Management der NOA-Architektur sind ebenfalls noch zu beantworten.
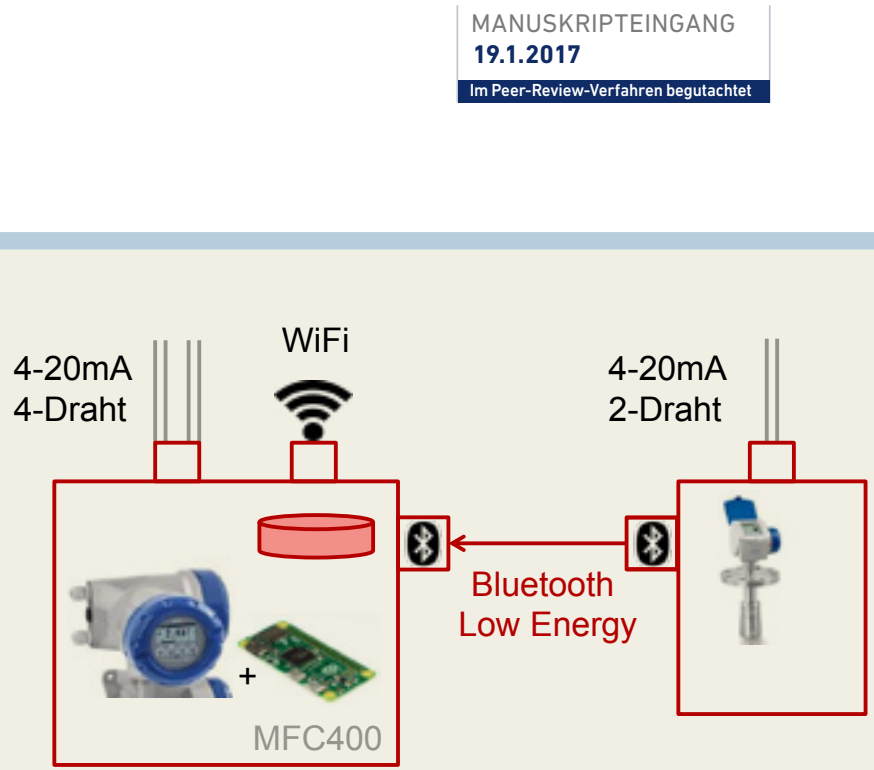

BILD 18: Anbindung von Zweileitergeräten 


\section{REFERENZEN}

[1] Diedrich, C., Hadlich, T. und Thron, M. (2015). Semantik durch Merkmale für 140. In: B. Vogel-Heuser, Hrsg., Handbuch Industrie 4.0. Berlin/Heidelberg: Springer

[2] Diedrich, C. und Riedl, M. (2015). Integration von Automatisierungsgeräten in Industrie-4.0Komponenten. In: B. Vogel-Heuser, Hrsg., Handbuch Industrie 4.0., Berlin/Heidelberg: Springer

[3] Diedrich, C., Bieliaiev, A., Bock, J., Gössling, A., Hänisch, R., Koziolek, H., Kraft, A., Pethig, F., Niggemann, O., Reich, J., Vollmar, F., Vialkowitsch, J. und Wende, J. (2016). Grammatik für Industrie 4.0-Komponenten. In: Tagungsband VDE-Kongress 2016. VDE-Verlag

[4] Diesner, M. (2016). MES im Internet of Things. [online] VDI-Z Integrierte Produktion. Verfügbar unter: http://www.ingenieur.de/VDI-Z/2016/Ausgabe-06/ Sonderteil-Industrie-4.0/MES-im-Internet-of-Things

[5] Epple, Ulrich. (2011). Merkmale als Grundlage der Interoperabilität technischer Systeme. at Automatisierungstechnik, 59(7), S. 440-450

[6] Graube, M., Pfeffer, J., Ziegler, J. und Urbas, L. (2012). Linked data as integrating technology for industrial data. International Journal of Distributed Systems and Technologies (IJDST), 3(3), S. 40-52

[7] Großmann, D., Banerjee, S., Bregulla, M., Braun, R. und Schulz, D. (2014). OPC UA Server Aggregation - The Foundation for an Internet of Portals. In: Proceedings of the 2014 IEEE Emerging Technology and Factory Automation, IEEE

[8] Großmann, D., Banerjee, S., Bregulla, M., Braun, R. und Schulz, D. (2014). Auf dem Weg zum Internet of Portals - Anwendungen für OPC UA Server Aggregation. atp edition, 56(7-8), S. 42-51

[9] Großmann, D., Braun, M., Danzer, B., Kaiser, A. und Riedl, M. (2015). FDI - Field Device Integration: Handbook for the unified Device Integration Technology. VDE Verlag

[10] Großmann, D. und Seintsch, S. (2016). FDI und OPC-UA: Grundsteine von Industrie 4.0. atp edition, 58(1-2), S. 34-39
[11] Herp, A. (2014). Industrie 4.0 - Was macht eine Revolution revolutionär?. [online] Markt \& Technik. Verfügbar unter: http://www.elektroniknet.de/ markt-technik/elektronikfertigung/was-macht-einerevolution-revolutionaer-113004.html

[12] IEC 62541-100 (2010). OPC UA Specification - Part 100, OPC UA for Devices, www.iec.ch

[13] IEC 62769-5 (2013). Field Device Integration (FDI), Technical Specification - Part 5: FDI Information Model. www.iec.ch

[14] Krauss, M., Pötter, T., latrou, C., Urbas, L. und Klettner, C. (2016). 100\% Wireless on Top. atp edition, 58(06), S. 50-65

[15] Kiesbauer, J., Banerjee, S., Erben, S. und Großmann, D. (2016). Predictive Maintenance auf der Basis von FDI und OPC UA. In: Tagungsband VDI-Kongress Automation 2016. VDI

[16] Menzel, G., Feier, D., Adam, G. und Hauff, T. (2003). Investitionssicherheit von Einrichtungen der Prozessleittechnik - eine (un)lösbare Aufgabe? atp edition, 45(3), S. 26-30

[17] NE 131 (2009). NAMUR-Standardgerät. http://www.namur.org

[18] Palm, F., Grüner, S., Pfrommer, J., Graube, M. und Urbas, L. (2015). Open source as enabler for OPC UA in industrial automation. In: 2015 IEEE 20th Conference on Emerging Technologies \& Factory Automation (ETFA), IEEE, S. 1-6

[19] Polke, M. (1985). Prozessleittechnik für die Chemie Status und Trend. atp-Sonderheft: NAMUR-Statusbericht 1985 „Prozessleittechnik für die chemische Industrie“. München: Oldenbourg, S. 5-14.

[20] Vogel-Heuser, B., Kegel, G. und Bender, K. (2009). Global Information Architecture for Industrial Automation. atp edition, 51(1-2), S. 108-115

[21] Vogel-Heuser, B., Diedrich, C. und Broy, M. (2013). Anforderungen an Cyber-Physical Systems vor dem Hintergrund unterschiedlicher Domänenmodelle der Automatisierungstechnik und der Informatik. at - Automatisierungstechnik, 61(10), S. 669-676

\section{AUTOREN}

Dipl.-Ing. CHRISTIAN KLETTNER (geb. 1980) absolvierte eine Ausbildung zum Prozessleitelektroniker bei BASF SE bevor er Automatisierungstechnik an der Hochschule Mannheim studierte. Bei der BASF arbeitet er im Senior Project BASF 4.0 und beschäftigt sich mit Digitalisierung im Produktionsumfeld. Innerhalb der Namur leitet er die Arbeitkreise 2.8
„Automatisierungsnetzwerke und Dienste“ und 4.15 „Mobile Automation“.

BASF SE, G-FTT/E,

67056 Ludwigshafen,

Tel. +49 (0) 6216043487 ,

E-Mail: christian.klettner@basf.com 


\section{AUTOREN}

Dr.-Ing. THOMAS TAUCHNITZ (geb. 1957) studierte in Hannover Elektrotechnik und promovierte im Bereich der Regelungstechnik. Bei Sanofi-Aventis Deutschland arbeitet er im Industrial Engineering for Emerging Markets. Er ist Vorstandsmitglied der Namur und Leiter des GMA-FA 6.16. Arbeitsschwerpunkte sind Prozess- und Betriebsleitebene sowie Engineering-Daten.

Prof. Dr.-Ing. ULRICH EPPLE (geb. 1953), war 1979 bis 1985 wissenschaftlicher Mitarbeiter am Institut für Systemdynamik und Regelungstechnik Universität Stuttgart, 1985 bis 1990 Mitarbeiter im Ressort Prozessleittechnik in der Bayer AG, 1991 bis 1995 Geschäftsführer der Gesellschaft für Prozesstechnik GmbH, und leitet seit 1995 den Lehrstuhl für Prozessleittechnik, RWTH Aachen. Hauptarbeitsgebiete: Modellbildung in der Leittechnik, formale Beschreibungsmethoden, Anwendung modellgetriebener Verfahren in der Prozessautomatisierung. Assistenzsysteme zur Unterstützung von Ingenieurprozessen.

M. Sc. LARS NOTHDURFT (geb. 1989) hat 2015 sein Studium der Automatisierungstechnik an der RWTH Aachen abgeschlossen. Seitdem arbeitet er als wissenschaftlicher Mitarbeiter am Lehrstuhl für Prozessleittechnik in Aachen. Sein Fachgebiet liegt beim automatisierten Austausch von Informationen durch Modelltransformationen im Umfeld von Industrie 4.0.

Prof. Dr.-Ing. CHRISTIAN DIEDRICH (geb. 1957) hat technische Kybernetik und Automatisierungstechnik studiert und lehrt und forscht an der Otto-vonGuericke-Universität und am Institut für Automation und Kommunikation (ifak) e.V. in Magdeburg. Seine Schwerpunkte liegen auf dem Gebiet der Automation in digital betriebenen Produktionssystemen basierend auf informations- und wissensbasierten Methoden. Dazu gehören vor allem die industrielle Kommunikation, Integrations- sowie Beschreibungsmethoden.Er leitet unter anderem den Arbeitskreis „Ontologie“ in der Plattform Industrie 4.0 und ist Convenor in der IEC-Strandardisierung von EDDL, FDT und FDI.

M. Sc. TIZIAN SCHRÖDER (geb. 1991) ist Mitarbeiter am Lehrstuhl Integrierte Automation an der Otto-von-Guericke-Universität Magdeburg. Zuvor studierte er Wirtschaftsingenieurwesen für Elektro- und Informationstechnik mit dem Schwerpunkt Automatisierungstechnik. Seine
Hauptarbeitsfelder sind semantische Beschreibungsmethoden als Grundlage für Interoperabilität in der digitalen Fabrik sowie Interaktionsmodelle für I4.0-Komponenten.

Prof. Dr.-Ing. DANIEL GROSSMANN (geb. 1977) studierte an der TU München Maschinenbau mit den Schwerpunkten Informationstechnik und Automatisierungstechnik. Nach seiner Promotion im Bereich Geräteintegration, für die er 2008 den Namur-Award erhielt, wechselte er in die Konzernforschung der ABB AG. Seit 2013 ist er Professor für Ingenieurinformatik und Datenverarbeitung an der Technischen Hochschule Ingolstadt. Zu seinen Forschungsthemen zählen industrielle Kommunikation, Geräteintegration und Informationsmodellierung in der Automatisierungstechnik als Basis für Industrie 4.0, Cyber Physical Systems und Industrial Internet of Things.

M. Sc. SUPRATEEK BANERJEE (geb. 1986) ist wissenschaftlicher Mitarbeiter am Zentrum für Angewandte Forschung der Technischen Hochschule Ingolstadt. Zuvor studierte er Informationstechnologie an der Universität Stuttgart. Sein Arbeitsgebiet umfasst die Informationsmodellierung sowie Kommunikationsarchitekturen für Industrie 4.0, Cyber Physical Systems und Industrial Internet of Things.

Dr. MICHAEL KRAUSS (geb. 1984) ist seit seiner Promotion in theoretischer Physik 2010 im Fachzentrum für Automatisierung der BASF SE in Ludwigshafen tätig. Seit 2013 leitet er ein Fachgebiet für Prozessleitsysteme mit dem Fokus auf Automatisierungsprojekten. In der NAMUR leitet er die Arbeitskreise 2.1 PLS und SPS und 4.20 Remote Operation.

Dipl.-Ing. CHRIS IATROU (geb. 1986) studierte Informationssystemtechnik mit Schwerpunkt auf digitalen VLSI-Schaltungsentwurf an der technischen Universität Dresden. Seit 2015 ist er wissenschaftlicher Mitarbeiter am Lehrstuhl für Prozessleittechnik der Technischen Universität Dresden. Seine Forschungsschwerpunkte liegen auf der Untersuchung von Automatisierungsarchitekturen zur Einbindung und dem steuerungsrelevanten Einsatz semantischer Daten in echtzeitkritischen Produktionsumgebungen.

Prof. Dr.-Ing. LEON URBAS (geb. 1965) ist Inhaber der Professur für Prozessleittechnik und Leiter der Arbeitsgruppe Systemverfahrenstechnik an der Technischen Universität Dresden. Er forscht an Methoden und Auswirkungen der digitalen Transformation in der Prozessindustrie. 